\title{
VARIABILIDAD INTERNA EN EL ALFARERO TEMPRANO DEL CENTRO-SUR DE CHILE: EL COMPLEJO PITRÉN EN EL VALLE CENTRAL DEL CAUTÍN Y EL SECTOR LACUSTRE ANDINO
}

\author{
INTERNAL VARIABILITY DURING THE EARLY CERAMIC PERIOD IN SOUTH- \\ CENTRAL CHILE: THE PITRÉN COMPLEX OF THE CENTRAL VALLEY OF \\ CAUTÍN RIVER AND THE ANDEAN LACUSTRINE SECTOR
}

Leonor Adán Alfaro ${ }^{1}$ y Rodrigo Mera Moreno ${ }^{2}$

\begin{abstract}
El presente artículo aborda la variabilidad del Alfarero Temprano en la zona Centro-Sur de Chile mediante la sistematización y comparación del registro arqueológico disponible en la sección media del valle del río Cautín y en los lagos Calafquén, Villarrica y Caburgua en ámbitos cordilleranos andinos, además de referencias a otras áreas trabajadas como la costa y ecosistemas insulares que permiten una discusión más integrada. Se aborda la variabilidad de dicha unidad arqueológica a partir de un análisis comparativo de los tipos, características y distribución de los asentamientos conocidos, sus materiales cerámicos y líticos, junto a otros indicadores materiales y biológicos. Lo anterior enfatiza los diferentes sustratos históricos, correspondientes al período arcaico, en los que se desarrollan los grupos adscribibles al Complejo Pitrén, postulándose diferentes procesos de integración y adopción de las innovaciones alfareras en la costa y en las zonas interiores. Se discute además las recurrentes dataciones tempranas y la existencia de expresiones alfareras iniciales diferentes a Pitrén. Se plantea la ocurrencia, en el marco de los estudios de complejidad social, de disímiles niveles de heterogeneidad en la estructura social de estas poblaciones alfarero tempranas y sus consecuentes expresiones espaciales y temporales. La relación con el alfarero tardío se interpreta a la luz del control y la forma en la toma de decisiones y la constitución de contextos sociales con mayor o menor presencia de dinámicas heterárquicas. Se destaca la necesidad del desarrollo de estudios que diversifiquen la mirada histórico-cultural que ha dominado la investigación regional para los períodos alfareros.
\end{abstract}

Palabras claves: Alfarero Temprano, Complejo Pitrén, organización social, Centro-Sur de Chile.

This article explores variability in Early Ceramic Period in the South-Central region of Chile through a systematization and comparison of the archaeological record from the the Middle Cautín River Valley and from the mountain lakes Calafquén, Villarrica and Caburgua, along with evidence from other regions such as the coast and island ecosystems which permits a more integrated comprehensive approach. The archaeological complex is specifically defined through a comparative analysis of settlement types, attributes, and distribution, including ceramic and lithic evidence, and other material and biological markers. This approach emphasizes the historical sequence beginning with the Archaic Period during which groups ascribed to the Pitrén Complex appear, indicating new processes of social integration and new ceramic innovations found in the coast and inlands. Recurrent early dates and other initial ceramic traditions are also discussed. The social structure of these early ceramic communities is defined as heterogeneous in light of their material spatial and temporal correlates. Their relationship with the Late Ceramic reflects a variation in control mechanisms, decision making, and the constitution of social contexts with more or less occurrence of heterarchic dynamics. Emphasis is placed on the need to employ approaches different from that of culture-history, which has dominated regional research for ceramic periods.

Key words: Early Ceramic Period, Pitrén Complex, social organisation, South-Central Chile.

Hacia fines de la década de los 80 se publicaban dos trabajos sintéticos sobre los períodos alfareros del Centro-Sur de Chile (Aldunate 1989; Dillehay 1990) en los cuales se proponía la existencia de los Complejos Pitrén y Vergel sobre la base de las primeras periodificaciones de Menghin (1962) ${ }^{1} \mathrm{y}$ estudios acotados a la zona del Cautín (Sánchez et al. 1981-82; Inostroza y Sánchez 1984; Gordon 1985). Tales trabajos significaron un aporte sustantivo para la comprensión de dichos complejos, funerarios en el caso de Aldunate y alfareros en el caso de Dillehay, señalando aspectos relativos a su distribución espacial y temporal, características de los cementerios y de sus conjuntos alfareros; con

1 Dirección Museológica, Universidad Austral de Chile. Casilla 586-Valdivia, Chile. ladan@uach.cl

2 O’Higgins 395. Gorbea, Región de La Araucanía, Chile. meragol@entelchile.net 
formulaciones que permitieron e impulsaron el desarrollo de la arqueología en la región, destacando la necesidad de profundizar las investigaciones de estas tempranas manifestaciones alfareras.

Su preocupación en el refinamiento de la historia cultural regional así como su adhesión a la naturaleza "formativa" de este primer complejo, enfatizó, no obstante, una perspectiva local y ecológica que permitió observar una posible variabilidad cultural temprana, ya que "si bien este complejo puede tener antecedentes septentrionales, probablemente se establece sobre el sustrato recolector previo, que representaría el poco conocido estadio arcaico local" (Aldunate 1989:339), como también cronológica, debido a que "No se sabe si Pitrén es realmente el complejo cerámico más temprano o solamente uno de los diferentes complejos contemporáneos presentes en distintas partes de la región Extremo Sur de Chile" (Dillehay 1990:107).

En este marco iniciamos el año 1993 el estudio de los períodos alfareros, en especial el Complejo Pitrén, primero a partir del análisis de colecciones y luego en sucesivas investigaciones en los sectores lacustres cordilleranos, lagos Calafquén, Villarrica y Caburgua, secciones de la cuenca del río Cautín y del río Valdivia, las que nos han permitido profundizar y explorar temáticas sobre las sociedades que ocuparon estos territorios durante el primer milenio de nuestra era (p.ej., Adán y Mera 1997a; Mera y Adán 2000; Adán y Alvarado 1999; Adán y Reyes 2000; Mera y Munita 2006; Reyes et al. 2003-2004) (Figura 1). A la par se desarrollaron otras investigaciones que ampliaron el conocimiento de este período en la cuenca del Cautín y en ámbitos costeros e insulares (Ocampo et al. 2004; Quiroz 1997; Quiroz y Sánchez 2005; Sánchez 1997). Tanto nuestras investigaciones, como aquellas desarrolladas en el valle medio del Cautín y en la costa septentrional, permitieron ampliar la estricta definición de complejo ${ }^{2}$, abordando diversos aspectos de la configuración social de las sociedades alfarero tempranas de la región Centro-Sur.

El trabajo que presentamos, en consecuencia, tiene como propósito general profundizar el estado del conocimiento sobre las sociedades alfarero tempranas del Centro-Sur de Chile. Para ello analizamos el registro arqueológico de dos áreas, el valle central del Cautín y la zona lacustre cordillerana en torno al volcán Villarrica, cuyas evidencias del Alfarero Temprano superan con creces los asentamientos aislados o únicos. Ambas áreas, no obstante, han sido trabajadas bajo diferentes enfoques teóricometodológicos con la consecuente disparidad en los resultados disponibles. En la discusión de algunas temáticas, incorporamos antecedentes de las zonas costeras y de cuencas fluviales más meridionales, que nos permiten un abordaje más integrador.

La aproximación que desarrollamos privilegia el análisis ya no de las regularidades del complejo -que ya rescataran las definiciones sintéticas que hemos mencionado- sino más bien la interpretación de las diferencias, de la variabilidad entre dos localidades y también aquellas a nivel de cada localidad (cfr. Hodder 1994:154-160, 168). Para ello efectuamos un análisis crítico y comparativo de los tipos, características y distribución de los asentamientos conocidos, sus materiales cerámicos y líticos, junto a otros indicadores materiales y biológicos. A partir de estos antecedentes, discutimos la naturaleza de la estructura social de estas poblaciones abordando los disímiles niveles de heterogeneidad social que vemos expresada en elementos del registro arqueológico, los cuales se comportan diferencialmente en el espacio y en el tiempo. Abordamos así, las evidencias de grupos adscribibles al Complejo Pitrén, y su relación con los sustratos histórico-regionales correspondientes al período Arcaico, postulando diferentes procesos de integración y adopción de las innovaciones alfareras en la costa y en las zonas interiores, como asimismo las recurrentes dataciones tempranas que evidenciarían la existencia de expresiones alfareras iniciales diferentes a Pitrén. Los momentos finales del Alfarero Temprano y su relación con el Alfarero Tardío se evalúan a partir de indicadores de diferenciación social y la constitución de contextos sociales con mayor o menor presencia de dinámicas heterárquicas (Crumley 1995).

Lo anterior nos permite presentar una visión actualizada de lo que hoy conocemos sobre las poblaciones alfarero tempranas en los bosques templados de la vertiente occidental andina, centrándonos en características y dinámicas de la estructura social de dichas poblaciones y en su variabilidad interna, configurando un panorama más comprensivo de estas sociedades y de la historia prehispánica regional.

\section{Registro Arqueológico en Dos Localidades}

Los registros arqueológicos que sistematizamos a continuación (Tabla 1) son resultados de programas y acciones de investigación diferentes en sus propósitos y alcances ${ }^{3}$. La perspectiva de análisis 
Tabla 1. Indicadores arqueológicos de las localidades de Cautín y la zona lacustre.

Archaeological indicators from the Cautín River locality and lacustrine area.

\section{Evidencias e \\ indicadores}

arqueológicos

Tipos de asentamientos registrados

Sitios de cementerio (Aldunate 1989; Dillehay 1990; Adán y Mera 1997; Mera y Adán 2000).
Sección lacustre (lagos Villarrica y Calafquén)

Sitios abiertos habitacionales, reparos rocosos y cementerios (Menghin 1962; Berdichewsky y Calvo 1972-73; Aldunate 1989; Adán y Mera 1997; Adán et al. 2001; Reyes et al. 2003-2004; Mera et al. 2010).

Situación espacial de Asociados a las cuencas fluviales a distancias los asentamientos variadas de los cursos de agua. Emplazamiento en lomajes con visibilidad al entorno y los volcanes y en sectores planos. (Sánchez et al. 1981-82; Inostroza y Sánchez 1984; Gordon 1984; Aldunate 1989; Dillehay 1990; Adán y Mera 1997; Mera y Adán 2000; Ocampo et al. 2004).
En terrazas lacustres a una cota aproximada de $300 \mathrm{msm}$ (ecotono actitudinal entre el bosque caducifolio y el laurifolio).

En valles interiores de las cuencas lacustres, en valles altos asociados a pasos cordilleranos y asociados a cursos fluviales o de agua menores.

(Adán et al. 2004)
Características de los cementerios
Cementerios de dimensiones variables, desde pequeños conjuntos a grandes complejos fúnebres. Visibilidad hacia los valles fluviales y en algunos casos a la Cordillera de los Andes. Fosas cavadas con ofertorios fúnebres, enterratorios simples y múltiples. Distanciados de los espacios domésticos.

Sobre los cuerpos se identificaron mujeres, niños y hombres; se observaron posiciones decúbito lateral y dorsal (Lof Mahuida y Licanco Chico). (Sánchez et al. 1981-82; Inostroza y Sánchez 1984; Gordon 1984; Aldunate 1989; Dillehay 1990; Adán y Mera 1997; Mera y Adán 2000; Ocampo et al. 2004)
Cementerios de dimensiones acotadas (p.ej., Pitrén), distanciados de los espacios domésticos.

Significativa visibilidad hacia el lago y el volcán.

Fosas cavadas con ofertorios fúnebres, enterratorios simples.

Sobre los cuerpos, se infiere una posición decúbito lateral izquierda con la cabeza orientada hacia el Este (Los Chilcos). (Adán y Mera 1997; Mera y Adán 2000; Alvarado 2000; Adán y Reyes 2000)

Conjuntos más heterogéneos y mayor número de modelados. (Adán y Mera 1997; Adán y Alvarado 1999) temente mono Alvarado 1999)

Producción alfarera local con predominio de pastas graníticas seguidas de las moscovitas. Conjuntos eminentemente monocromos, con formas restringidas con cuellos cilíndricos o hiperboloides, restringidas de cuello y perfil inflectado y vasijas abiertas. (Reyes et al. 2003-2004; Donoso y Gallegos 2008).

Escasa presencia de ofrendas líticas en cementerios. Los sitios habitacionales registran toda la cadena operativa con el dominio de materias primas locales y en menor frecuencia obsidiana, riolita y calcedonia; se registran también artefactos de molienda. (Mera y Becerra 2002; García 2007; García 2007; Mera 2008; Cordero 2010).
Conjuntos líticos fúnebres y domésticos
Artefactos líticos de uso doméstico como parte de las ofrendas funerarias. Se registran piezas para molienda, sobadores, raspadores, cepillos, cantos astillados, lascas con retoque y para el caso de Villa JMC-1, guijarros aplanados de lava basáltica. Destaca también el registro de posibles torteras, en Lof Mahuida, elaboradas sobre esquisto.

No se conocen materiales líticos de sitios habitacionales. (Gordon 1986; Ocampo et al 2004; Mera y Munita 2008). 
Continuación Tabla 1

\begin{tabular}{l}
$\begin{array}{c}\text { Evidencias e } \\
\text { indicadores } \\
\text { arqueológicos }\end{array} \quad$ Valle del Cautín $\quad$ Sección lacustre (lagos Villarrica y Calafquén) \\
\hline
\end{tabular}

Antecedentes En Licanco Chico, se infieren períodos de estrés bioantropológicos nutricional y una dieta propia de una economía de caza recolección con un incipiente aporte de productos ricos en hidratos de carbono. Los restos dentales documentan abrasión plana indicación del consumo de elementos fibrosos escasos en partículas.

Se observa deformación craneana intencional, en algunos individuos de sexo masculino de tipo tabular erecto occipital o vértico occipital. (Aspillaga y Retamal 2001 en Ocampo et al. 2004).

Conjuntos No se conocen estudios de arqueofauna.

En Los Chilcos-1, se documentan lesiones asociadas a períodos de estrés nutricional en la niñez y la inclusión en la dieta de alimentos ricos en hidratos de carbono y la presencia de chipping. Domina el desgaste en semicopa asociado al consumo de materiales blandos. (Alfonso 2000 en Adán y Reyes 2000). arqueofaunísticos
No se conocen estudios de arqueofauna.

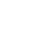

\begin{abstract}
Se observa una continuidad de las prácticas de cazarecolección registradas desde el período Arcaico, documentada a partir de restos líticos y evidencias de arqueofauna.

La existencia de artefactos óseos en la larga secuencia ocupacional del sitio comprometido da cuenta también de estos elementos de continuidad. (Velásquez y Adán 2004; García 2005; García 2006a, García 2006b; García 2007; Mera 2008; Cordero 2010).
\end{abstract}

Manejo de recursos vegetales
Consumo de alimentos ricos en hidratos de carbono que podrían ser tanto silvestres como cultivados.

Ofrendas de artefactos de molienda. (Aspillaga y Retamal 2001 en Ocampo et al. 2004; Mera y Munita 2008).
El estudio de piezas dentales reconoce el consumo de alimentos ricos en hidratos de carbono (Los Chilcos). En el mismo sitio se identificó en el contenido de vasijas Chenopodium sp. y Galium sp.

Se han reconocido semillas comestibles como Bromus mango/unioloides y dos tipos de Poaceae y otras ingresadas con ítems comestibles como Aristotelia chilensis, Gevuina avellana, Podocarpaceae y Peumus boldus (Marifilo-1, nivel alfarero).

Variado uso de taxa como recurso dendroenergético (Marifilo-1, nivel alfarero).

Se reconoce el uso de materiales líticos sobre materiales blandos que corresponderían a maderas y artefactos de molienda (Marifilo-1, Loncoñanco-2, Los Resfalines-1, Flor del Lago-1).

Restos de maíz en el sitio Villarrica W10 datados hacia el siglo IX d.C.

(Alfonso 2000 en Adán y Reyes 2000; Lehnebach et al. 2007; Mera y Becerra 2002; García 2007; Mera 2008; Cordero 2010).

No se han reconocido hallazgos sobre textilería y metalurgia.

Evidencia de metalurgia en cobre como parte ofertorio fúnebre de, al menos, cinco individuos en el sitio Villa JMC -1 en Labranza. Evidencia del uso de textiles, al menos como parte de uno de los contextos funerarios de Villa JMC-1. Se registra un fragmento de textil con fibras torcidas elaborado en pelo de camélido (alpaca). (Mera y Munita 2008; Bracchitta y Seguel 2009).
Otros indicadores materiales y prácticas tecnológicas
No se han documentado dataciones tan tempranas en comparación con la costa y la cordillera, sin embargo presenta una mayor recurrencia de fechados en momentos intermedios (500-1.000 d.C.).
Se extendería desde antes de la era cristiana, hasta momentos tardíos (ca. 1.000 d.C.). 


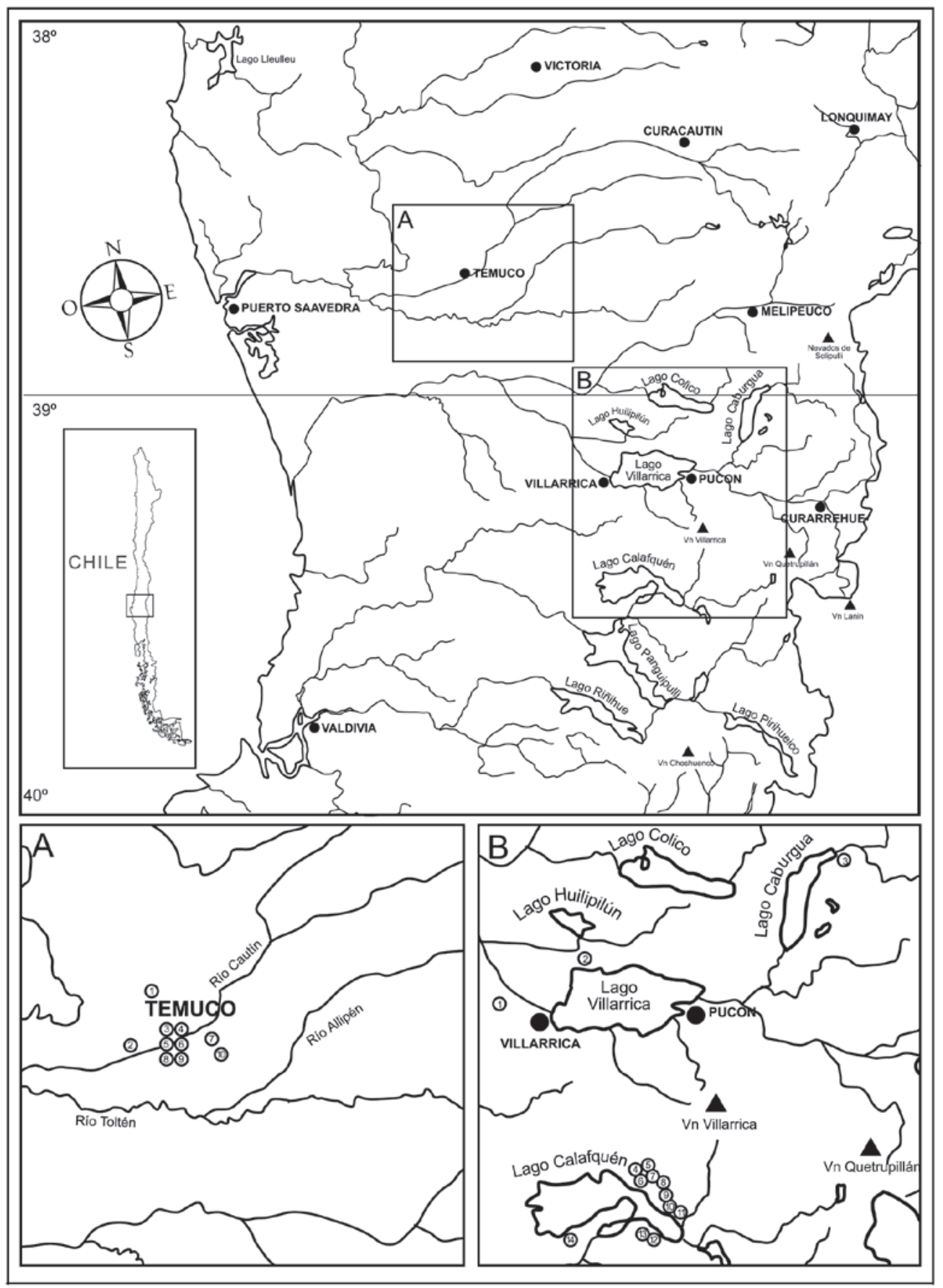

Figura 1. Sitios del Complejo Pitrén mencionados en el texto. (A) Sección media del valle del Cautín. (1) Huimpil, (2) Villa JMC-1, (3) Shell Norte, (4) Industria Bandag, (5) Liceo Industrial, (6) Campus Andrés Bello, (7) Lof Mawida-Km 15, (8) Padre Las Casas, (9) Maquehue y, (10) Licanco Chico-Km 20. (B) Sector lacustre cordillerano. (1) Lliu-Lliu-1, (2) Flor del Lago-1, (3) Playa Negra-1, (4) Challupén, (5) Challupén (VM), (6) Los Chilcos, (7) Antilef-1, (8) Alero Ñilfe-1, (9) Alero Marifilo-1, (10) Alero Loncoñanco 2-3, (11) Alero Los Resfalines-1, (12) Musma-2, (13) Musma-1 y (14) Pitrén.

Pitrén Complex settlements mentioned in the text. (A) Middle Cautín River Valley. (1) Huimpil, (2) Villa JMC-1, (3) Shell Norte, (4) Industria Bandag, (5) Liceo Industrial, (6) Campus Andrés Bello, (7) Lof Mawida-Km 15, (8) Padre Las Casas, (9) Maquehue and (10) Licanco Chico-Km 20. (B) Andean lakes. (1) Lliu-Lliu-1, (2) Flor del Lago-1, (3) Playa Negra-1, (4) Challupén, (5) Challupén (VM), (6) Los Chilcos, (7) Antilef-1, (8) Alero Nilfe-1, (9) Alero Marifilo-1, (10) Alero Loncoñanco 2-3, (11) Alero Los Resfalines-1, (12) Musma-2, (13) Musma-1 and (14) Pitrén. 
que desarrollamos no puede soslayar dificultades planteadas a la interpretación en consideración al estado de la investigación, por lo cual algunas formulaciones deben considerarse a nivel hipotético. Sin perjuicio de lo anterior, planteamos un ejercicio analítico, crítico y comparativo ${ }^{4}$, que tiene como propósito aportar a la comprensión de la variabilidad al interior de esta unidad arqueológica y la forma en que se expresan la heterogeneidad y la desigualdad social en la estructura social de dichas poblaciones (Blau 1977; Mc Guire 1983), en un intento por avanzar más allá del estricto enfoque histórico-cultural que ha dominado la discusión arqueológica al respecto.

\section{El ámbito de los lagos cordilleranos del eje Villarrica-Lanin}

La llamada "región del Calafquén" (Berdichewsky y Calvo 1972-73: 529) fue objeto de una primera inspección con el objeto de desarrollar una mejor definición del Complejo Pitrén a nivel de localidad, agregando luego nuevos sitios en los lagos Villarrica y Caburgua (Figura 1).

A la fecha, se han efectuado trabajos arqueológicos y se conocen dataciones absolutas para más de una decena de sitios correspondientes a cementerios, aleros y sitios a "cielo abierto". Ellos se localizan en sectores circundantes a los lagos Calafquén, Villarrica y Caburgua. La sistematización ofrecida por los resultados de prospecciones efectuadas en la zona ha permitido la identificación de un conjunto significativo de sitios alfarero tempranos, a juzgar por la presencia de rasgos diagnósticos del material cerámico (Munita y Adán 2009).

En su conjunto, tanto las diversas clases de asentamiento como la frecuencia de sitios alfarero tempranos dan cuenta de una ocupación efectiva y constante articulando un patrón de asentamiento propio de los espacios lacustres (Adán et al. 2004:1447-1453). Estos sitios se emplazan próximos o muy próximos a la costa; en terrazas lacustres más altas; en pequeños valles y quebradas interiores; en terrazas fluviales y en sectores cordilleranos altos, como Curarrehue, en cotas entre los 200 y $1.450 \mathrm{msm}$, lo que representa una importante variabilidad ambiental.

El estudio de la alfarería fragmentada correspondiente a sitios abiertos del lago Calafquén (Reyes et al. 2003-2004:160-164) permitió la identificación de 32 patrones de pastas agrupados en tres familias: granítica, muscovita y volcánica-granítica. La distribución de estas familias es bastante homogénea en los distintos sitios, con dominancia de las pastas graníticas seguida de las muscovitas. Los áridos del antiplástico son de origen autóctono, lo que evidenciaría producción alfarera local. El conjunto es mayoritariamente pulido, aunque también se registran fragmentos engobados rojos, blancos y cafés, con improntas de hojas, bícromos rojo sobre blanco y negro sobre rojo, en técnica negativa. El análisis de otros sitios habitacionales abiertos, Flor del Lago-1, Villarrica W8, Playa Negra y Liucura-1, ubicados en la zona del lago Villarrica y Caburgua, informan de un panorama similar. Se identificaron conjuntos eminentemente monocromos con piezas restringidas con cuellos cilíndricos o hiperboloides, restringidas de cuello y perfil inflectado y vasijas abiertas (Donoso y Gallegos 2008).

La alfarería recuperada de los sitios de cementerios conocidos: Pitrén, Challupén $(\mathrm{VM})^{5}$, Challupén-2, Pucura, Traitraico, Los Chilcos y Lliu-Lliu-1, informa de conjuntos en los que dominan las piezas monocromas. Formalmente se reconoce la mayor popularidad de los jarros y la presencia recurrente de ollas, botellas, escudillas y tazas. Entre las diferencias, se observa la presencia de un jarro asa mango en Los Chilcos, tipo ausente en los otros contextos y en Challupén-2 una mayor proporción de modelados. Las piezas con decoración en técnica negativa negro-rojo están presentes en Challupén-2, Challupén (VM)y Pitrén, y corresponden a la variedad A en disposición radial. También se conocen excepcionales modelados con representaciones antropomorfas y zoomorfas en Challupén-2, Traitraico y Pitrén (Berdichewsky 1972-73; Menghin 1962).

El material lítico, por su parte, ha sido estudiado a partir de los restos recuperados de sitios abiertos y aleros que han sido sondeados y/o excavados: Antilef-1, Rinconada Challupén, Alero Ñilfe-1, Marifilo-1, Loncoñanco 2-3, Los Resfalines-1, Rilul-1, Flor del Lago-1, Liucura-1, Playa Negra-1 (Mera y Becerra 2002; García 2005; García 2006a; Mera et al. 2007; García 2007; Mera 2008; Cordero 2010). Entre las clases tipológicas recuperadas se identificaron núcleos, restos de desbaste, artefactos no formatizados con rastros complementarios (ANFRC, sensu Aschero 1975) y otros formatizados. En todos los sitios, como es de esperar, la mayor abundancia corresponde al material de desbaste (derivados y desechos de talla), seguido de los ANFRC. La mayor 
frecuencia y diversidad de materiales líticos está registrada en los sitios abiertos de Playa Negra-1 y Flor del Lago-1. Entre las materias primas usadas dominan las locales, andesita y basalto porfírico, en menor frecuencia se registran el basalto afanítico, y luego las alóctonas como la obsidiana, la riolita, el cristal de roca y la calcedonia ${ }^{6}$. Entre estas últimas domina la obsidiana, en sus variedades negra, café y traslúcida, con porcentajes muy similares, cercanos al 25\%, para Flor del Lago-1 y Playa Negra-1. Entre los ANFRC se identifica toda la variedad tipológica propia de contextos habitacionales, centrados en las tareas de cortar-raer, cortar-cepillar, cortarraspar y triturar. Por su parte, entre los materiales formatizados se reconocieron puntas de proyectil, raspadores y artefactos de molienda (morteros y manos). Hasta ahora hemos podido identificar que algunas variedades de la obsidiana recuperada de Flor del Lago-1 y Playa Negra-1 provienen de la zona de Llaima-Lonquimay, lo que evidenciaría la circulación de esta materia prima durante este período y su obtención por parte de las poblaciones de los entornos lacustres ya sea por movilidad o intercambio (Stern et al. 2009).

Los estudios de arqueofauna han sido practicados sobre restos recuperados del sitio Marifilo-1 y Loncoñanco 2 (Velásquez y Adán 2004; García 2005). Entre las taxas identificadas se cuenta zorro chilla (Pseudalopex griseus), pudú (Pudu pudu), colo colo (Felis colo colo), chingue (Conepatus chingue) y aves estacionales. Destaca la presencia de Artiodactila mayor, presumiblemente huemul (Hippocamelus bisulcus), espécimen hoy extinto en la zona. En el registro arqueofaunístico del sitio Marifilo-1 se observa una importante continuidad con los niveles arcaicos; existe un aprovechamiento continuo del pudú desde el Arcaico temprano hasta el Alfarero, mientras que el zorro chilla está representado desde el Arcaico medio hasta el Alfarero. Una situación similar es la que se observa en la manufactura de artefactos (Velásquez y Adán 2004; García 2006b), con la presencia en los niveles alfareros de una espátula-punzón sobre tibia distal de huemul y un fragmento de punzón, similares a los de los niveles tempranos, dando cuenta de un conocimiento tradicional e importante conservadurismo.

Indicadores adicionales, provistos por el análisis de restos humanos (dentales) en Los Chilcos, documentan la presencia de caries y abrasión en grado moderado, que se habría producido por ingesta de productos ricos en carbohidratos de consistencia blanda y pegajosa, junto a la ocurrencia de chipping e hipoplasia, asociada a una lesión relacionada con períodos de estrés nutricional en la niñez (Alfonso 2000 en Adán y Reyes 2000: 33).

Por último, para casos particulares, contamos con antecedentes arqueobotánicos significativos respecto de las prácticas de subsistencia y del ambiente. En primer lugar, se cuenta con el estudio de carporrestos de vasijas de funebria también para Los Chilcos, donde se informa de Chenopodium sp., Galium sp., cyperáceas y gramíneas sin identificar (Belmar y Quiroz en Adán y Reyes 2000). La presencia de esta quínoa silvestre, asociada a los datos bioantropológicos, constituiría antecedentes indirectos de la existencia de prácticas hortícolas en el Alfarero Temprano de los sectores lacustres. En la costa NW del lago Villarrica, por otro lado, recientes hallazgos en el sitio Villarrica W10 han permitido datar fehacientemente maíces de un fogón de un sitio abierto hacia los 890-1020 DC 7 (Tabla 2) (Mera et al. 2009).

Un panorama más completo, aunque nuevamente acotado a un sitio particular, es el que ofrece el estudio antracológico y carpológico de Marifilo-1. En este asentamiento, el conjunto de restos vegetales analizados (Lehnebach et al. 2008) informa de una amplia variedad de taxa identificadas durante los períodos alfareros, en comparación con los niveles holocénicos medios y tempranos. Se identificaron semillas comestibles y otras no comestibles (Lehnebach et al. 2007, Tabla 2). Entre las primeras destaca Bromus mango/unioloides, también presente en los niveles arcaico medio, Pisum sativa, que daría cuenta de la disturbación del contexto y probablemente granos del género Chusquea. Entre las segundas se registra cf. Aristotelia chilensis, cf. Peumus boldo, cf. Gevuina avellana. La perspectiva antracológica, por su parte, confirma la mayor variedad de taxa en los períodos alfareros distinguiendo una distribución similar entre los carbones dispersos en el alero y aquellos recuperados de fogones, señalando un uso oportunístico de las maderas, como leña entre otros.

\section{La sección media de la cuenca del río Cautín}

A diferencia del ámbito cordillerano, el derrotero de la investigación desarrollada en esta localidad ha seguido caminos diferentes. Hasta la fecha no se ha desarrollado ninguna investigación de carácter sistemático o que trascienda la eventualidad del 
Tabla 2. Dataciones absolutas de sitios del Complejo Pitrén y otros. Absolute dates for Pitrén Complex settlements and others.

\begin{tabular}{|c|c|c|c|c|c|}
\hline Sitio & $\begin{array}{c}\text { Edad a.p./Edad } \\
\text { radiocarbónica } \\
\text { convencional }\end{array}$ & $\begin{array}{l}\text { Fecha/Edad en años } \\
\text { calendario }\end{array}$ & Tipo y n en lab. & Referencia Bibliográfica & Área Geográfica \\
\hline Alero Quino-1 & $1.865+/-200$ & 130 d.C. & UCTL-823 & Quiroz et al. 1997 & \multirow{3}{*}{ Valle Central Norte } \\
\hline Alero Quino-1 & $1.740+/-170$ & 255 d.C. & UCTL-824 & Quiroz et al. 1997 & \\
\hline La Tereña & $1.255+/-110$ & 740 d.C. & UCTL-884 & Adán y Mera 1997 & \\
\hline Campus A. Bello & $1.620+/-170$ & 375 d.C. & UCTL-886 & Adán y Mera 1997 & \multirow{6}{*}{$\begin{array}{l}\text { Sección Media de } \\
\text { Cautín }\end{array}$} \\
\hline Shell Norte & $1.490+/-150$ & 505 d.C. & UCTL-883 & Adán y Mera 1997 & \\
\hline Huimpil & $1.290+/-80$ & 660 d.C. & GIF-4948 & Gordon 1984 & \\
\hline Licanco Chico (Km 20) & $1.110+/-60$ & 890 d.C. & AZ-12240 & Ocampo et al. 2004 & \\
\hline Villa JMC-1 & $1.060+/-40$ & 940 d.C. & Beta-241265 & Mera y Munita 2008 & \\
\hline Lof-Mawida (Km 15) & $830+/-135$ & 1.170 d.C. & AZ-12241 & Ocampo et al. 2004 & \\
\hline Pichipehuenco-2 & $2.190+/-40$ & 380-160 a.C. & Beta 273150 & Adán et al. 2010b & \multirow{7}{*}{$\begin{array}{l}\text { Sector cordillerano } \\
\text { septentrional }\end{array}$} \\
\hline Sector Callaqui (Queuco) & $2.140+/-215$ & 135 a.C. & $\mathrm{TL}$ & Bustos 2009 & \\
\hline Sector Reserva (Bío Bío) & $1.955+/-195$ & 50 a.C. & $\mathrm{TL}$ & Bustos 2009 & \\
\hline Sector Trapa Trapa (Queuco) & $1.805+/-180$ & 200 d.C. & $\mathrm{TL}$ & Bustos 2009 & \\
\hline Sector Butalelbún (Queuco) & $1.695+/-155$ & 310 d.C. & $\mathrm{TL}$ & Bustos 2009 & \\
\hline Sector El Barco (Bío Bío) & $1.390+/-140$ & 615 d.C. & $\mathrm{TL}$ & Bustos 2009 & \\
\hline Sector Reserva (Bío Bío) & $1.060+/-110$ & 945 d.C. & $\mathrm{TL}$ & Bustos 2009 & \\
\hline Flor del Lago-1 & $2.110+/-40$ & 340-40 a.C. & Beta- 253960 & Adán et al. 2010b & \multirow{19}{*}{$\begin{array}{l}\text { Lacustre } \\
\text { cordillerano }\end{array}$} \\
\hline Flor del Lago-1 & $2.080+/-40$ & 190 a.C. -10 d.C. & Beta-273148 & Adán et al. 2010b & \\
\hline Villarrica W10 & $1.080+/-40$ & 890-1020 d.C. & Beta-273151 & Adán et al. 2010b & \\
\hline Los Chilcos & $1.650+/-170$ & 350 d.C. & UCTL-1235 & Adán y Reyes 2000 & \\
\hline Challupén-2 & $1.540+/-160$ & 455 d.C. & UCTL-892 & Adán y Mera 1997 & \\
\hline Alero Marifilo-1 & $1.480+/-50$ & 520 d.C. & UCTL-1278 & Reyes et al. 2003-2004 & \\
\hline Alero Nilfe & $1.420+/-140$ & 580 d.C. & UCTL-1453 & Reyes et al. 2003-2004 & \\
\hline Challupén-2 & $1.365+/-110$ & 635 d.C. & UCTL-1524 & Reyes et al. 2003-2004 & \\
\hline Alero Nilfe & $1.110+/-100$ & 890 d.C. & UCTL-1236 & Reyes et al. 2003-2004 & \\
\hline Los Lagos & $1.080 \pm 110$ & 915 d.C. & UCTL-885 & Adán y Mera 1997 & \\
\hline Escuela Collico-1 & $1.045+/-100$ & 960 d.C. & UCTL-1746 & Mera y Munita 2006 & \\
\hline Playa Negra-1 & $1.070+/-40$ & 890-1.030 d.C. & Beta-227246 & Adán et al. 2010b & \\
\hline Antilef-1 & $1.010+/-100$ & 990 d.C. & UCTL-1457 & Reyes et al. 2003-2004 & \\
\hline Pitrén & $1.000+/-100$ & 1.000 d.C. & UCTL-887 & Adán y Mera 1997 & \\
\hline Antilef-1 & $970+/-100$ & 1.030 d.C. & UCTL-1458 & Reyes et al. 2003-2004 & \\
\hline Musma-1 & $920+/-90$ & 1.080 d.C. & UCTL-1279 & Reyes et al. 2003-2004 & \\
\hline Antilef-1 & $920+/-90$ & 1.080 d.C. & UCTL-1279 & Adán y Reyes 2000 & \\
\hline Musma-1 & $735+/-50$ & 1.265 d.C. & UCTL-1276 & Adán y Reyes 2000 & \\
\hline Alero Rilul-1 & $680+1-60$ & 1.320 d.C. & UCTL-1277 & Reyes et al. 2003-2004 & \\
\hline
\end{tabular}


Continuación Tabla 2

\begin{tabular}{|c|c|c|c|c|c|}
\hline Sitio & $\begin{array}{c}\text { Edad a.p./Edad } \\
\text { radiocarbónica } \\
\text { convencional }\end{array}$ & $\begin{array}{c}\text { Fecha/Edad en años } \\
\text { calendario }\end{array}$ & Tipo y n en lab. & Referencia Bibliográfica & Área Geográfica \\
\hline P25-1 (Isla Mocha) & $1.940+/-180$ & 10 d.C. & Gd-9197 & Sánchez 1997 & \multirow{19}{*}{ Costa Norte } \\
\hline Talcahuano 1 & $1.845+/-100$ & 130 d.C. & UCTL-1051 & Quiroz 2010 & \\
\hline P25-1 (Isla Mocha) & $1.760+/-130$ & 190 d.C. & Gd-10007 & Sánchez 1997 & \\
\hline Lenga 2 & $1.570+/-160$ & 430 d.C. & UCTL-1630 & Quiroz 2010 & \\
\hline P10-1 (Isla Mocha) & $1.560+/-150$ & 430 d.C. & UCTL-537 & Quiroz 2010 & \\
\hline Huechicura 1 (Cañete) & $1.465+/-40$ & 520 d.C. & UCTL-1023 & Quiroz 2010 & \\
\hline LE-38 (Quinahue) & $1.325+/-130$ & 670 d.C. & UCTL-825 & Quiroz 2010 & \\
\hline P25-1 (Isla Mocha) & $1.310+/-130$ & 680 d.C. & UCTL-536 & Quiroz 2010 & \\
\hline Tranaquepe 1 & $1.275+/-100$ & 720 d.C. & UCTL-822 & Quiroz 2010 & \\
\hline P22-1 (Isla Mocha) & $1.250+/-100$ & 740 d.C. & UCTL-543 & Sánchez 1997 & \\
\hline P25-1 (Isla Mocha) & $1.240+/-130$ & 750 d.C. & UCTL-535 & Quiroz 2010 & \\
\hline P22-1 (Isla Mocha) & $1.210+/-130$ & 780 d.C. & UCTL-542 & Sánchez 1997 & \\
\hline P5-1 (Isla Mocha) & $1.210+/-110$ & 880 d.C. & Beta 73674 & Sánchez 1997 & \\
\hline P22-1 (Isla Mocha) & $1.200+/-140$ & 890 d.C. & Beta-71646 & Sánchez 1997 & \\
\hline P21-1 (Isla Mocha) & $1.060+/-100$ & 930 d.C. & UCTL-541 & Sánchez 1997 & \\
\hline P21-1 (Isla Mocha) & $1.030+/-110$ & 960 d.C. & UCTL-540 & Sánchez 1997 & \\
\hline P21-1 (Isla Mocha) & $1.020+/-100$ & 970 d.C. & UCTL-539 & Sánchez 1997 & \\
\hline P21-1 (Isla Mocha) & $1.010+/-100$ & 980 d.C. & UCTL-530 & Quiroz 2010 & \\
\hline Lonkotripay & $930+1-80$ & 1.065 d.C. & UCTL-1024 & Sánchez 1997 & \\
\hline Alero Ollita Encantada (40-70) & $1.205+/ 120$ & 800 d.C. & UCTL-1850 & Adán et al. 2007 & \\
\hline Fundo Santa María & $1.150+/-120$ & 845 d.C. & UCTL-888 & Adán y Mera 1997 & \\
\hline Alero Morro Gonzalo (70-80) & $1.055+/-100$ & 950 d.C. & UCTL-1855 & Adán et al. 2007 & \\
\hline Cueva Morro Gonzalo (60-110) & $980+/-95$ & 1.025 d.C. & UCTL-1856 & Adán et al. 2007 & Costa Sur \\
\hline Alero Ollita Encantada (0-30) & $965+/-90$ & 1.040 d.C. & UCTL-1849 & Adán et al. 2007 & \\
\hline Panitao-1 & $1.230+/-90$ & 775 d.C. & UCTL-1997 & Bustos 2009 & \\
\hline Panitao-1 & $1.155+/-100$ & 850 d.C. & UCTL-1998 & Bustos 2009 & \\
\hline
\end{tabular}

salvataje, lo que ha incidido en que la mayoría de los sitios corresponda a cementerios (Figura 1). Tal situación hace necesario mantener cautela con las comparaciones posibles.

En la década de los 80 además de Huimpil se realizaron numerosos rescates de cementerios y contextos funerarios en varios puntos de la ciudad de Temuco. La recuperación de todos ellos correspondió más bien a rescates y gracias al desarrollo de proyectos posteriores centrados en el estudio de las colecciones pudimos datar mediante TL varios de aquellos (Tabla 2).

Otros contextos como Huimpil, Licanco Chico (Km 20), Lof Mawida (Km 15) y Villa JMC-1 corresponden a yacimientos de mayores dimensiones en cuanto a superficie involucrada, número de contextos y piezas recuperadas. Este hecho podría tener un correlato en el patrón de asentamiento, en el sentido de que durante Pitrén la sección media de la cuenca del Cautín habría sido preferentemente ocupada por estos grupos (Figura 1).

Respecto de la alfarería, los trabajos de rescate y análisis realizados en los sitios rescatados en el By-Pass de Temuco permitieron agregar tres nuevas variedades: pichi-metawe, botellas con asas de suspensión y decoración modelada en el cuerpo de la pieza, además de jarros simétricos con modificaciones en el labio formando un sector 
acuminado o "vertedero" (Ocampo et al. 2004). Cabe destacar también el registro de una pipa cerámica en Licanco Chico-Km 20 (Ocampo et al. 2004) y 2 en Villa JMC-1 de Labranza (Mera y Munita 2008). Piezas inéditas hasta esta fecha en contextos funerarios, aunque sí observadas en las colecciones regionales.

Respecto del material lítico, en Huimpil se reparó en la presencia de una pieza lítica, correspondiente a una mano de moler fracturada, por lo que ha sido a partir de estos últimos trabajos que se ha puesto un mayor énfasis en la observación y descripción de estos restos. En los sitios del By-Pass se registraron artefactos de uso doméstico: cantos astillados de andesita, torteras elaboradas sobre esquisto, artefactos toscos sobre lascas, guijarros pulidos, raspadores, cepillos, sobadores y manos de moler (Ocampo et al. 2004). En tanto en Villa JMC-1 se registró la presencia de una punta de proyectil pedunculada sobre obsidiana "ahumada", un raspador sobre obsidiana "atigrada", algunos cantos astillados sobre andesita y basalto (similares a los del By-Pass) y la presencia en varios contextos de conjuntos con números variables de guijarros aplanados volcánicos, sin huellas aparentes de uso, pero que estaban claramente depositados como ofrendas mortuorias. Lo anterior se relaciona con la eventual movilidad de estos grupos y la circulación de materias primas. La obsidiana que se registra, muy probablemente procede del ámbito cordillerano, del área de aprovisionamiento de Melipeuco, como ha sido documentado en otros yacimientos arqueológicos de la cuenca del río Cautín, como en el alero Quillén-1 y en los sitios abiertos Tres Arroyos-1 y Los Riscos-1 (Stern et al. 2009). El hecho de estar presente en estos contextos permite atisbar la importancia que tuvo el espacio cordillerano para estos grupos del valle. Mención aparte merecen la ocurrencia de torteras sobre esquisto presentes en Lof Mawida-Km 15 por la implicancia indirecta que tendría en relación con la actividad textil y el manejo de los camélidos. Al respecto, cabe consignar el hallazgo de un pequeño trozo de textil elaborado con fibras torcidas de alpaca (Bracchitta y Seguel 2009) conservado gracias a las características biocidas del cobre a partir del cual fue elaborado uno de los aros recuperados en Villa JMC-1 (Mera y Munita 2008).

A diferencia del sector lacustre, los contextos del Cautín cuentan con mayor registro y variedad bioantropológica. Se ha recuperado evidencia en
Lof Mahuida-Km 15 (Ocampo et al. 2004) y Campus Andrés Bello (Sánchez et al. 1981-82) y destacan los casos de Licanco Chico-Km 20 (Ocampo et al. 2004) y Villa JMC-1, como los únicos que poseen restos humanos completos. De los análisis realizados, en Licanco Chico se registró la presencia de deformación craneana intencional sobre individuos masculinos, del tipo tabular erecto occipital o vértico occipital, lo cual de acuerdo a los autores vincularía a estas poblaciones con las de la zona central y Norte Chico donde también se ha registrado esta clase de deformación en poblaciones alfarero tempranas (Aspillaga y Retamal en Ocampo et al. 2004). A partir del análisis de los restos dentales se infiere una economía de caza y recolección con un incipiente aporte de productos ricos en hidratos de carbono, lo que se evidencia en la abrasión plana con escaso trauma oclusal de algunas piezas dentales.

Otros objetos registrados en sitios asociados a la cuenca del Cautín y que marcan una diferencia no sólo con el sector lacustre, sino con el resto de áreas o localidades son los aros de cobre recuperados como parte del ajuar funerario de algunos de los contextos del cementerio Villa JMC-1 (Mera y Munita 2008). $\mathrm{Su}$ registro es importante no sólo porque resultan los más tempranos de la región Centro-Sur, sino también porque demuestran que la ocurrencia de metales se presentaría desde Pitrén y no sólo desde El Vergel (cfr. Campbell 2004: 83), abriendo una interesante temática sobre la incorporación de esta nueva tecnología. Las características del estilo decorativo y probablemente tecnológico de las piezas recuperadas señalan conexiones estilísticas con piezas recuperadas de contextos Diaguitas y con otras en la provincia de la Pampa (Berón y González 2005), sin posibilidades de determinar a la fecha si ellas fueron producidas localmente o son expresión de una red amplia de interacción y movilidad.

\section{Discusión}

La situación descrita mediante el registro arqueológico de dos localidades, junto a antecedentes relevados en investigaciones practicadas en otras áreas como la cuenca del río Valdivia, la Isla Mocha y la costa de Arauco que añadimos como datos específicos para presentar una discusión más completa, nos permiten presentar una reflexión integradora de la situación del período Alfarero Temprano en la región Centro-Sur del país. 


\section{Situación cronológica y cultural}

En relación con aspectos cronológicos y culturales, el conjunto de dataciones disponibles (Tabla 2) señala la identificación del Complejo Pitrén desde al menos el siglo IV d.C., tanto en el valle medio del Cautín, ejemplificado en Shell Norte, como en las regiones lacustres, como lo evidencia Los Chilcos. En la costa, en tanto, se ha informado de "componentes alfareros estilísticamente vinculados al Complejo Pitrén, especialmente la presencia de fragmentos con pintura negativa" (Quiroz 1997:240). Los sitios alfareros de la Mocha presentan dataciones entre los 10 d.C. ${ }^{8}$ y 1680 d.C. (Sánchez 1997) con una presencia más recurrente desde los siglos VII y VIII d.C. (Quiroz y Sánchez 2005:371). Otros cementerios, adscribibles al Complejo Pitrén y presentes en ámbitos fluviales de valles costeros, lo constituyen Tranaquepe y Loncotripay, este último datado hacia el siglo XI d.C. En la costa de Valdivia se han documentado sitios de alero tanto hacia el sur como hacia el norte de la Bahía de Corral entre el 950 d.C. y 1.450 d.C. (Adán et al. 2007), mientras que hacia el interior se conocen los cementerios Fundo Santa María y Los Lagos, entre los siglos IX y X d.C. (Adán y Alvarado 1999; Adán y Mera 1997a).

En suma, el registro indica la existencia de cementerios adscribibles al Complejo Pitrén tanto en el valle del Cautín como en la zona lacustre cordillerana desde el 300 d.C., mientras que en la costa y sectores insulares las fechas más tempranas se presentan desde el 430 d.C. en P10-1 (Sánchez 1997:129). En la costa septentrional continental particularmente, se ha planteado la evidencia de ocupaciones Pitrén entre los años 400 d.C. a 900 d.C., tanto para cementerios como sitios habitacionales al sur del río Lebu. Además se registra otro conjunto denominado Complejo Temprano, diferente a Pitrén, representado por los sitios Lenga-2 y El Arenal-1, documentando para la zona entre el Bío-Bío y la península de Arauco, al norte del Lebu, la existencia de un complejo diferente y contemporáneo a Pitrén (Quiroz et al. 2010:448).

En una medida importante, lo anterior tiene relación con el sustrato previo, correspondiente al período Arcaico, sobre el cual se reconocen luego las poblaciones alfareras. $\mathrm{Al}$ respecto, en una reciente síntesis de las ocupaciones alfareras de la cuenca del río Valdivia (Adán et al. 2007) hemos enfatizado el reconocimiento de las diferencias de las ocupaciones arcaicas tanto en ámbitos costeros como cordilleranos, para sugerir la participación de las poblaciones alfarera tempranas de los espacios lacustres cordilleranos de una Tradición Arqueológica de Bosques que mantendría prácticas tradicionales, particularmente económicas y vinculadas a los modos de subsistencia con base en momentos arcaicos (Adán et al. 2007; Adán et al 2010). A la par, los estudios de Navarro en la costa han caracterizado un modo de vida típicamente costero asociado a la amplia tradición de puntas foliáceas, articulándose principios de territorialidad desde el Arcaico Medio (Navarro y Pino 1999; Navarro 2004).

En este escenario hemos sugerido que el cambio cultural que define el Alfarero Temprano en relación con el período previo, particularmente aquel que genéricamente vinculamos al Complejo Pitrén, con el entierro de sus difuntos en cementerios familiares o comunitarios, ocupación de diferentes clases de asentamiento, economías mixtas, adopción de un estilo tecnológico alfarero fuertemente pautado, entre otros, ocurriría de distinta manera en los diferentes espacios ecológicos.

$\mathrm{Al}$ respecto, y particularmente para el caso de la cuenca de Valdivia en la que queda incluido el lago Calafquén, hemos planteado que las poblaciones interiores incorporarían las innovaciones alfareras, de manera más homogénea y más tempranamente, en términos globales, que las poblaciones de la costa (Adán et al. 2007); ello considerando las dataciones con las que contamos, en especial aquellas de sitios de cementerio, y la definición de la Tradición Arqueológica de Bosques como un modo de vida fuertemente adaptado a los bosques templados, pero con evidentes restricciones alimentarias (Catalán 1999; Godoy 2010) y por tanto, teóricamente, más abiertos a la innovación, en comparación con las poblaciones costeras con prácticas de caza-recolección eficientes y conservadoras (Navarro 2004). La identificación de nuevos complejos alfareros distintos a Pitrén, tal como ha sido descrito en la zona de Arauco (Quiroz et al. 2010), señala que en la costa septentrional existirían igualmente condiciones económicas y culturales que harían a ciertos grupos incorporar dicha innovación a su forma de vida, en un contexto en que las poblaciones cazadoras-recolectoras tradicionales representarían el sistema económico 
social dominante, coexistiendo con otras que comienzan a distanciarse de éstas y que, probablemente, sean igualmente diferentes de lo que entendemos clásicamente como Pitrén.

Lo anterior requiere obviamente mayor sustento empírico, pero apunta hacia una situación de mayor complejidad y variaciones locales en la transición del período Arcaico al Alfarero, de lo que hasta ahora podíamos vislumbrar.

Evidentemente, un tema vinculado a la discusión previa es el conjunto de dataciones tempranas con que contamos hasta la fecha y que originalmente se calificaron como "intrusivas" (Valdés et al. 1982:133). En la actualidad, sin embargo, ellas son recurrentes en diferentes tipos de sitios, ambientes, materiales ${ }^{9}$ y métodos de datación (Quiroz et al. 1997; Sánchez 1997; Valdés et al. 1982). Por ahora podemos señalar la efectiva ocurrencia de estas ocupaciones iniciales en esta variedad de contextos, cuyo futuro estudio permitirá, además de la caracterización sistemática de sus registros materiales y una mejor definición de las mismas, de manera como se ha establecido para Chile Central (Sanhueza y Falabella 19992000), abordar la forma en que los diversos grupos de cazadores recolectores del período Arcaico incorporan la innovación alfarera. Como antecedentes disponibles a la fecha debe mencionarse el Complejo Temprano antes mencionado, definido por Quiroz y colaboradores como “ocupaciones alfareras más tempranas definidas ya hace algunos años como ocupaciones de pueblos portadores de cerámica sin agricultura (Seguel y Campana 1970)" (Quiroz et al. 2010:448). Por otra parte, nuestras investigaciones en la zona cordillerana nos han permitido identificar dataciones tempranas en el sitio abierto Pichipehuenco-2 en pinalerías de la zona de Lonquimay y en el sitio Flor del Lago-1, también abierto-habitacional, en la costa norte del lago Villarrica en la que hemos testeado su datación temprana, hacia el 100 a.C., mediante dos fechados, al que se agrega un tercero en un nivel sin cerámica cercano al 800 a.C., haciendo coherente la secuencia y, evidente la reiteración en la ocupación del mismo lugar por parte de poblaciones que transitan hacia la adopción de la alfarería desde tempranos momentos (Tabla 2) (Adán, García, Mera et al. 2010).

Respecto de la relación de Pitrén con el Complejo Vergel y la aparición de cerámica Valdivia, son evidentes las dificultades y el "amarre" interpretativo que ha impuesto el marco histórico cultural y tipológico en la interpretación de la historia prehispánica regional a la par de los provechosos resultados que ha permitido. En el caso del Villarrica y el Calafquén, la inexistencia de los clásicos enterratorios en urna de El Vergel, ha estimulado la discusión de la temática en otros términos, a lo cual se agrega la propuesta de Aldunate sobre una fase oriental y tardía del Complejo Pitrén ${ }^{10}$ que consideráramos para interpretar el registro alfarero con fechas hasta ca. los 1.300 d.C. tardías en el Calafquén como un correlato occidental de la fase oriental descrita (Adán et al. 2004). Los estudios que siguieron han planteado que en el caso del Calafquén, el Alfarero Tardío presentaría rasgos característicos de tradiciones cerámicas tardías del valle central y la cordillera, como la pintura roja sobre engobe blanco, y carecería de otras como las estrías anulares ${ }^{11}$ y las asas con protúberos, conformando un componente cultural diferente, más cercano a lo que se ha definido como Complejo Cerámico Pitrén (Becerra y Reyes 2005:395) ${ }^{12}$. Centrándose en sitios de alero de la misma localidad, se ha planteado, adicionalmente, que en algunos casos, particularmente Marifilo-1, Curilef-1 y Puente Seco se observarían características de la cerámica más claramente tardías y que, en tal caso, es probable la coexistencia de grupos de distintas tradiciones (Mera et al. 2007), y microvariaciones en la producción cerámica (Reyes 2009), aspecto al que volveremos más adelante.

La pregunta evidente, en el caso de la región cordillerana, es cómo entender estos contextos alfareros con dataciones tardías, ante lo cual la estricta filiación a complejos cerámicos resulta completamente insuficiente así como perspectivas generalistas que ocultan la importante variabilidad ocurrida hacia el 1.100-1.500 d.C. en la prehistoria regional.

En el caso del Cautín, por otra parte, se han identificado enterratorios en urnas claramente adscribibles al Complejo El Vergel en sitios como Padre Las Casas (Gordon 1978) y Quinta Santa Elvira (Adán y Mera 1997b; Sánchez e Inostroza 1984), ambos datados hacia mediados del siglo XIII. Sobre la forma en que ocurre el cambio cultural que supone el registro de asentamientos Vergel en el valle medio de esta cuenca fluvial, no existen suficientes antecedentes ni hipótesis publicadas a nivel de esta localidad; se asume, más 
bien, que este territorio participaría del área de extensión del Complejo El Vergel y de los cambios tecnológicos, en el patrón de asentamiento, modo de vida y sistema de creencias que ello supondría (cfr. Campbell 2004; Dillehay et al. 2007; Dillehay 2007; Aldunate 2005) ${ }^{13}$.

\section{Variabilidad social, materialidad y asentamiento}

Las dos materialidades mejor conocidas, cerámica y lítica, permiten una más detallada caracterización del complejo y la comprensión de su diversidad interna.

En el caso de la cerámica de funebria, pese a las diferencias espaciales y cronológicas que hemos descrito con anterioridad, los conjuntos definen un estilo común, al menos en lo formal ${ }^{14}$, sin grandes diferenciaciones e innovaciones. Esta adhesión formal, no obstante, resulta con diferentes grados de pericia técnica como se refleja comparando tres distintos cementerios de la cuenca del río Valdivia, que en conjunto avalan la idea de producción local. Los conjuntos de los cementerios Pitrén, en el Calafquén, Escuela Collico-1 en las inmediaciones de Loncoche y Los Lagos en la ciudad del mismo nombre, situados cronológicamente entre los siglos IX y XI presentan en el primer y tercer caso similitudes en la variedad de formas modeladas y decoradas (Adán y Alvarado 1999; Adán y Mera 1997a), además de una alta calidad en la manufactura, mientras que en Collico se registraron junto a vasijas de buena factura, otras especialmente toscas, que evidencian eventos de aprendizaje y cierta variabilidad en la práctica, competencias y resultados de los artesanos (Mera y Munita 2006:64). Los ocupantes de Escuela Collico-1, emplazados en un sector de espesos estompamientos vinculados al río Cruces, dan cuenta de la apropiación diferencial del estilo tecnológico alfarero Pitrén y de su posición social en el espacio social fúnebre construido regionalmente ${ }^{15}$. De esta manera, las poblaciones que portan cerámica de este estilo y se entierran con un patrón compartido acceden y se apropian contemporáneamente de manera diferencial de los procedimientos técnicos y códigos simbólicos reflejados en la cerámica que ofrendan a sus muertos, dando cuenta de una participación diferencial en los procesos de aprendizaje, esferas de interacción y circulación tanto de recursos como de conocimientos. Se añade además que el conjunto tipológico, en términos de las variedades formales registradas, identificado en Collico se asemeja a la primera clase de asociaciones de tipos formales caracterizada por la dominancia de tipos monocromos y la presencia en momentos tempranos (Adán y Mera 1997a).

Para el caso del Calafquén, el estudio de la fragmentería cerámica, particularmente las pastas, considerado uno de los elementos relevantes en la definición de un "estilo tecnológico" (Falabella y Sanhueza 2005-2006:115-116), permitió la identificación de tres patrones de pastas, compuestas de áridos mayoritariamente "de origen autóctono, denotando un uso de materias primas y producción local" (Reyes et al. 2003-2204:163). Los diversos tipos de pastas se usan indistintamente para diferentes formas y se distribuyen homogéneamente en los distintos sitios analizados lo que, empleando conceptos aplicados en la zona central, daría cuenta de un estilo tecnológico característico del Calafquén. Adicionalmente, un estudio efectuado sobre material cerámico de sitios datados con fechas tardías de los lagos Villarrica y Calafquén bajo el concepto de estilo tecnológico (Reyes 2008), señala nuevamente el predominio de las familias graníticas, pero se reconocen tres sitios, Puraquina en Villarrica y Loncoñanco 2 y 3 en Calafquén en los que aumentan notablemente las pastas micáceas. En estas se reconocen además microvariaciones que señalan en el caso del Calafquén patrones que no aparecen en Villarrica (Reyes 2009). Lo anterior indicaría que en un conjunto de sitios del Villarrica y Calafquén, datados con posterioridad al 1.000 d.C. la alfarería acusa prácticas de aprovisionamiento diferenciados que homologa sitios de ambas cuencas lacustres configurando un primer conjunto en los que dominan las pastas graníticas y otros en los que éstas y las de la familia mica presentan frecuencias similares. Estas "microvariaciones" apuntarían, desde nuestro punto de vista, a la definición de grupos distintos en cuanto a la producción cerámica, en los cuales los segundos añaden un nuevo patrón más típicamente tardío, mientras que los primeros se encuentran más vinculados con lo que se ha definido para el Complejo Pitrén.

En el caso de los materiales líticos, habría que distinguir que -según lo conocido hasta ahora- se observan diferencias marcadas entre los conjuntos líticos rescatados y el tipo de sitio al que se 
asocian. En efecto, para el ámbito cordillerano, en aquellos asentamientos en que se han registrado niveles tempranos es posible distinguir claras diferencias entre los sitios abiertos y los contextos en alero. Los primeros, correspondientes a sitios habitacionales, dan cuenta de conjuntos más bien monocomponentes donde es posible observar prácticamente toda la cadena operativa y en los que luego de las materias primas locales existe una preponderancia del uso de la obsidiana (cercana al 25\%), situación que daría cuenta del nivel de movilidad y acceso a sectores más septentrionales, dentro del mismo ambiente cordillerano, tal como se ha documentado a partir del análisis de proveniencia de restos líticos de estas materias primas (Stern et al. 2009). También se observa la presencia de artefactos formatizados y con huellas de uso que evidenciarían buena parte de las actividades que se realizan en los contextos habitacionales, asociadas especialmente al consumo, procesamiento y alimentación del grupo. En tanto los sitios de alero manifiestan en primer término una reiterada ocupación de los asentamientos, lo que altera la integridad estratigráfica de los mismos y la contextualización posible de obtener para cada período y, en segundo término, la observación de la preponderancia de las estrategias oportunistas y expeditivas como se ha descrito.

Para la localidad del Cautín, también veríamos diferencias importantes, que más bien manifestarían el tipo de investigación desarrollada y donde, hasta la fecha, se desconocen los sectores habitacionales. Los contextos recuperados corresponden a sitios funerarios en los que veríamos más bien las expresiones simbólicas del manejo y uso del recurso lítico. Se registran, en todo caso, artefactos de molienda, de caza y otros que denotan parte de las actividades desarrolladas. Un rasgo observado sería la recurrencia en cuanto a la presencia de cantos astillados o guijarros asociados a ciertas materias primas como andesitas celestes y basaltos de grano fino, además de guijarros aplanados de lava volcánica.

Respecto de las clases de asentamientos, una que podemos explorar, por contar con datos comparativos, es la magnitud de los sitios de cementerio, de menores dimensiones en el caso del lago Calafquén, tanto por la superficie que éstos comprometen como por la cantidad de ofrendas totales y para cada contexto funerario, y grandes sitios fúnebres en el caso del Cautín (Tabla 1).
Estos sitios, contemporáneos, permiten suponer, en el caso del Cautín, la existencia de formas sociales de congregación que superan la unidad familiar. En el Calafquén, en cambio, los cementerios corresponderían a unidades sociales más acotadas o pequeñas, probablemente familias extendidas, con una distribución más segmentada espacial y socialmente ${ }^{16}$, situación que no parece variar ostensiblemente en términos cronológicos (p.ej., Los Chilcos-1, datado hacia el siglo IV, y Pitrén, en el siglo X). Lo anterior expresaría una opción cultural de estas poblaciones lacustres vinculada a sus condiciones de existencia, sociales, económicas y geográficas, que resulta y se articula con el paisaje cultural por ellos habitado.

El nodo de articulación social que señalan los sitios del By-Pass, Huimpil y Villa JMC-1 de Labranza, supone estrategias de ordenamiento social elaboradas y actualizadas por un amplio grupo social en cada evento fúnebre. En este sentido, debe notarse, pese a su obviedad, que el Cautín se organiza en un entorno fluvial, recordando las sociedades ribereñas o leufuche propuesta para tiempos históricos (Bengoa 2003:52, 56; Boccara 1989:77) ${ }^{17}$, mientras que en el Calafquén se trata de una cuenca lacustre, dominada por un volcán y con pinalerías cercanas, en la que la movilidad posible debió articular y favorecer rutas lacustres (Carabias et al. 2007), complementadas en segundo término por vías terrestres y fluviales.

El sistema y características de los escasos sitios habitacionales, identificados hasta ahora en el entorno lacustre, y que lamentablemente aún no conocemos en el caso del Cautín, podría relacionarse con el nivel de segmentación que expresan los sitios de cementerio y con la mantención de una modalidad de asentamiento más cercana a la de grupos cazadores-recolectores, con sitios abiertos de escasa densidad material, un conjunto de aleros en los que se reconocen contextos domésticos y pequeños cementerios en topos (locus) privilegiados para acoger a los muertos.

Con todo, se observa como una característica común tanto de los cementerios del Cautín como aquellos de los lagos, la selección de lomajes con importante visibilidad del entorno, en especial de los volcanes, que da cuenta del reconocimiento de sistemas de creencias compartidas vinculadas a una forma de asentarse y habitar los territorios. La orientación de los cuerpos inferida a partir de los restos humanos encontrados tanto en Los 
Chilcos, en el lago Calafquén, como en Licanco Chico en el Cautín, señala una orientación de los cuerpos en dirección $\mathrm{N}-\mathrm{S}$, con el cráneo hacia el sur, en posición decúbito lateral derecho y la mirada hacia el oriente (Adán y Reyes 2000; Ocampo et al. 2004), lo que da cuenta de una evidente intencionalidad y refuerza la idea del vínculo y relevancia del ámbito cordillerano en el sistema de creencias de estas comunidades, a lo que se agrega además, en el caso del Cautín, la depositación de guijarros aplanados de lava volcánica como ofrendas funerarias ${ }^{18}$.

\section{Dinámicas sociales: hacia una comprensión de los procesos de complejización social}

El registro arqueológico, que hemos descrito comparativamente, da cuenta de la importante variabilidad interna del Complejo Pitrén, que entendemos expresa procesos de complejización social ocurridos en las sociedades alfareras tempranas de los bosques templados del Cono Sur de América. Los modelos histórico-culturales y ecológico-culturales que hemos privilegiado en el estudio de estas poblaciones prehispánicas creemos que pueden ser enriquecidos centrándonos en los procesos de interacción social y el rol activo de la cultura material, bajo la perspectiva teórica, amplia y debatida, de los estudios de complejidad social ${ }^{19}$.

Nuestro acercamiento plantea que la complejidad social no debe ser entendida mecánicamente como expresión exclusiva de procesos de jerarquización social o como una categoría dada en la que sólo ciertas sociedades complejas, como opuestas a simples, pueden ser incluidas (Mc Guire 1983; Souvatzi 2007; Wynne-Jones y Kohring 2007). Superando la visión taxonómica que ofrecen los esquemas tipológicos neoevolucionistas (p. ej., Flannery 1975; Fried 1985; Sahlins 1981) ${ }^{20}$, es posible abordar la temática de la estructura social (Blau 1977) observando las diferencias y variaciones presentes en el asentamiento y en la distribución y comportamiento de parte de la cultura material y su relación con la heterogeneidad y la desigualdad, dos variables básicas para abordar el estudio de la complejidad social (Crumley 1995; Mc Guire 1983).

De esta manera, observamos que las significativas variaciones en los cementerios, sobre todo en la envergadura de éstos, dan cuenta de una mayor heterogeneidad en la zona del Cautín a partir de los siglos VIII y IX, que expresaría en primer lugar la existencia de comunidades más amplias y con ello la consecuente mayor probabilidad de diferentes roles y estatus (Crumley 1995). Lo anterior se evidencia además en la presencia de ciertos rasgos materiales que distinguen a individuos enterrados como el número de vasijas ofrendadas, ceramios modelados excepcionales y piezas únicas hasta ahora, como torteras, collares y aros de cobre. Para el caso de estos últimos, su registro en el sitio Villa JMC-1 en Labranza constituye un dato significativo puesto que por tratarse de un bien escaso evidenciaría el acceso diferencial a ciertos recursos, bienes o grupos, y la apertura a innovaciones tecnológicas que sólo se popularizarán más tarde, durante el Alfarero Tardío prácticamente en toda la Araucanía (Campbell 2004). Junto a lo anterior destaca la ocurrencia de la deformación craneana que refuerza la idea de cierta heterogeneidad social y quizá su actuación como un marcador identitario ${ }^{21}$.

En la misma lógica, las asociaciones de tipos cerámicos en los conjuntos fúnebres que observamos tanto en el Cautín como en la zona lacustre con dataciones entre los siglos IV y IX correspondiente a la primera clase de asociaciones (Adán y Mera 1997a), daría cuenta de contextos menos heterogéneos, comunes en ambos territorios en fechas tempranas y que permanecen hasta momentos tardíos como lo hemos descrito para Escuela Collico-1 en un sector más meridional. En tiempos más tardíos, por otra parte, aumenta la proporción de piezas decoradas mediante modelado y decoración rojo-negro en técnica negativa; aparecen además representaciones antropomorfas, ausentes en los contextos más tempranos, que denotan un cambio representacional, por cuanto la figura humana adquiere un lugar en el contexto simbólico de la funebria, antes desconocido.

Como se ha destacado, la relación entre heterogeneidad y desigualdad no es necesariamente positiva, vale decir, que no necesariamente covarían (Mc Guire 1983; Souvatzi 2007) y cada caso debe evaluarse en su contexto. Por lo mismo, el establecimiento de menor heterogeneidad en el caso de la zona lacustre, evidenciada en una menor concentración demográfica, debe entenderse en un contexto en que la distribución de los asentamientos y por tanto la ocupación de espacio es más segmentada espacialmente. Adicionalmente, la ocurrencia de vasijas modeladas con representaciones zoomorfas 
y antropomorfas, indicador que en la localidad tiene mayor representación proporcional que en los cementerios del Cautín, significa que pese a la menor envergadura de los asentamientos, es más común el empleo de estrategias de distinción de ciertos individuos en los contextos fúnebres, lo cual vemos expresado en la inclusión de estas piezas excepcionales de elaborada y experta manufactura.

Lo anterior señala que en diversos cementerios de los contextos lacustres, la existencia de individuos diferenciados o desiguales es más frecuente. Siguiendo a Crumley (1995), las ocupaciones tardías de los lagos expresarían, a juzgar por su menor densidad demográfica, una menor heterogeneidad o diversidad en los roles y estatus, aunque con algunos de éstos claramente distinguidos (desiguales o diferentes) en cuanto expresan en la materialidad alfarera significativos códigos simbólicos y el acceso a bienes cuya manufactura requirió la aplicación de conocimiento experto y una significativa inversión de labor (capital cultural y económico, sensu Bourdieu 1999), lo que, al menos en el ámbito de lo fúnebre, los acerca más a estructuras jerárquicas que aquellas heterárquicas descritas para el Cautín.

La expresión desigual de esas diferencias es un problema que puede ser abordado en las maneras en que se incorporan nuevos elementos culturales del Alfarero Tardío. En el valle medio del Cautín, como en buena porción de la Araucanía septentrional, se ha planteado una temprana incorporación de elementos correspondientes al Complejo El Vergel, que en cuanto tecnologías dan cuenta de cambios sustanciales en el modo de vida (cfr. Aldunate 2005; Campbell 2004; Dillehay et al. 2007). En cambio, en el caso de la zona lacustre, particularmente en el Calafquén, se ha sostenido la persistencia de poblaciones alfarera tempranas o al menos su coexistencia con otras más tardías (Aldunate 1989; Becerra y Reyes 2005; Hajduk 1986; Mera et al. 2010). Tal apertura a las innovaciones en el caso del Cautín ocurriría y sería posible en un contexto heterogéneo con escasa desigualdad, en el cual, consecuentemente, la toma de decisiones estaría menos controlada y centralizada (McGuire 1983:107). La desigualdad en este caso pareciera operar en un patrón más heterárquico (Crumley $1995,2007)$ en el que convergen y gravitan un conjunto de posiciones sociales competentes de manera diferencial o interrelacionada en la toma de decisiones ${ }^{22}$.

En el caso del lago Calafquén, en cambio, operarían dinámicas de control más eficientes sobre la adopción de innovaciones -dentro de la lógica conservadora que se asume para Pitrén, que bien puede ser entendida también como un proceso de "arcaísmo"23-, resultando una dinámica social en que los cambios se generan en un escenario menos heterogéneo y con mayor desigualdad hacia momentos tardíos del Alfarero Temprano, comparado a lo descrito para el Cautín, a otro, en la primera mitad del segundo milenio, que señala la coexistencia, y probablemente competencia, de grupos de distinta filiación cultural cohabitando un mismo territorio.

\section{Consideraciones Finales}

La sistematización hasta acá presentada informa de la variabilidad interna del Complejo Pitrén a partir de una perspectiva enfocada en el asentamiento, la organización social y la expresión material. La comparación entre dos localidades permite un panorama más diverso de lo que se ha definido como la unidad arqueológica más destacada del período Alfarero Temprano regional. Lo anterior necesariamente debe ampliarse con nuevas investigaciones y con la integración de otras localidades, como la costa y la zona oriental andina. Las formulaciones presentadas buscan diversificar la mirada teórica con la que hemos venido estudiando las sociedades alfareras prehispánicas de territorio tradicionalmente mapuche, fuertemente apegadas a la historia cultural o a las perspectivas ecológicas y tipológicas, enfatizando una comprensión de las diferencias presentes en el registro arqueológico desde una perspectiva de las dinámicas sociales asociadas a las estrategias económicas y uso del vasto territorio regional.

Agradecimientos: Investigación realizada en el marco del proyecto Fondecyt 1060216 "Habitando bosques, lagos y volcanes: comparación de las ocupaciones arcaico y alfarero temprano en los ámbitos cordilleranos Llaima-Lonquimay y Villarrica-Lanin (39S)". Agradecemos la lectura crítica y observaciones de los evaluadores del manuscrito que han permitido mejorar sustantivamente el presente artículo. 


\section{Referencias Citadas}

Adán, L. y M. Alvarado

1999 Análisis de colecciones alfareras pertenecientes al Complejo Pitrén: una aproximación desde la arqueología y la estética. Actas de las III Jornadas de Arqueología de la Patagonia, 245-268. Universidad Nacional del Comahue e INAPL. Neuquén-Buenos Aires.

Adán, L., C. García y R. Mera

2010 La tradición arqueológica de bosques templados y su estudio en la región lacustre cordillerana de las regiones IX y X. Actas del XVII Congreso Nacional de Arqueología, Tomo 2, pp. 1461-1471. Sociedad Chilena de Arqueología, Universidad Austral de Chile. Ediciones Kultrún, Valdivia.

Adán, L., C. García, R. Mera, M. Godoy, D. Carabias y D. Munita

2010 Informe Final Proyecto Fondecyt 1060216. Valdivia. Manuscrito en posesión de los autores.

Adán, L. y R. Mera

1997a Acerca de la distribución espacial y temporal del Complejo Pitrén. Una revaluación a partir del estudio sistemático de colecciones. Boletín Sociedad Chilena de Arqueología 24:33-37.

1997b Anexo Nº1. La Tradición Cerámica Bícroma rojo sobre blanco en la región centrosur: los estilos Vergel y Valdivia. Propuesta Tipológica Morfológica-Decorativa de la Alfarería. Estudio morfológico de las urnas El Vergel. Informe Proyecto Fondecyt 1950823. Archivo Nacional, Santiago. Manuscrito en posesión de los autores.

Adán, L., R. Mera, F. Bahamondes y S. Donoso

2007 Síntesis arqueológica de la cuenca del río Valdivia: proposiciones a partir del estudio de sitios alfareros prehispánicos e históricos. Revista Austral de Ciencias Sociales 12:5-30.

Adán L. y V. Reyes

2000 Sitio Los Chilcos. Descripción y análisis de un nuevo cementerio Pitrén en la región del Calafquén. Boletín de la Sociedad Chilena de Arqueología 30:30-40.

Adán, L., V. Reyes y R. Mera

2004 Ocupación humana de los bosques templados del Centro-Sur de Chile. Proposiciones acerca de un modo de vida tradicional. Actas del IV Congreso Chileno de Antropología, Tomo II, pp. 1444-1445. Colegio de Antropólogos de Chile, Universidad de Chile, Santiago.

Aldunate, $\mathrm{C}$.

1989 Estadio alfarero en el sur de Chile. En Culturas de Chile. Prehistoria, desde sus Orígenes hasta los Albores de la Conquista, editado por J. Hidalgo, V. Schiappacasse, H. Niemeyer, C. Aldunate e I. Solimano, pp. 329-348. Editorial Andrés Bello, Santiago.

2005 Presentación del Simposio El Complejo Arqueológico El Vergel en el Centro-Sur de Chile: relaciones temporales y espaciales. Actas del XVI Congreso Nacional de Arqueología, pp. 329-336. Museo de Historia Natural de Concepción y Sociedad Chilena de Arqueología. Ediciones Escaparate, Concepción.

Alvarado, $\mathrm{M}$.

2000 Vida, muerte y paisaje en los bosques templados. Un acercamiento a la estética del paisaje en la Región del Calafquén. Aisthesis 33:198-216.
Aschero, C.

1975 Ensayo para una clasificación morfológica de artefactos líticos aplicada a estudios tipológicos comparativos. Informe al CONICET. Buenos Aires. Manuscrito.

Becerra, M. y V. Reyes.

2005 Análisis contextual de sitios alfareros tardíos de la localidad de Calafquén, precordillera andina de la IX y X regiones. Actas del XVI Congreso Nacional de Arqueología, pp. 389-398. Museo de Historia Natural de Concepción y Sociedad Chilena de Arqueología. Ediciones Escaparate, Concepción.

Bechis, M.

1989 Los lideratos políticos en el área Araucano-Pampeana en el siglo XIX: ¿autoridad o poder? Ponencia presentada en el I Congreso de Etnohistoria Argentina, Buenos Aires.

Bengoa, J.

2003 Historia de los Antiguos Mapuches del sur. Desde antes de la Llegada de los Españoles Hasta las Paces de Quilin. Catalonia, Santiago.

Berdichewsky, B. y M. Calvo

1972-73. Excavaciones en cementerios indígenas de la región de Calafquén. Boletín de Prehistoria, Número Especial. Actas del VI Congreso de Arqueología Chilena, 529-558. Museo de La Serena y Sociedad Chilena de Arqueología, La Serena.

Berón, M. y L. González

2005 Análisis de composición de adornos metálicos de un contexto funerario de cazadores-recolectores. El sitio Chenque I, provincia de La Pampa. Actas del Primer Congreso Argentino de Arqueometría, en prensa.

Blau, P.

1977 Inequality and Heterogeneity: A Primitive Theory of Social Structure. The Free Press, Nueva York.

Boccara, G.

2007 Los Vencedores. Historia del Pueblo Mapuche en la Época Colonial. Línea editorial IIAM, Universidad Católica del Norte y Universidad de Chile, San Pedro de Atacama, Santiago.

Bourdieu, P.

1985 The social space and the genesis of groups. Social Science Information 24(2):195-220. SAGE, London, Beverly Hills and New Delhi.

1999 [1994] Razones Prácticas. Sobre la Teoría de la Acción. Editorial Anagrama, Barcelona.

Bracchitta, D. y R. Seguel

2009 Informe de Intervención. Estudio e intervención de los materiales arqueológicos provenientes del sitio Villa JMC-01, Labranza. Temuco, IX Región de La Araucanía. Manuscrito en posesión de los autores.

Burke, P.

1996 Venecia y Amsterdam. Editorial Gedisa, Barcelona.

Bustos, V.

2009 Consideraciones Cronológicas a la luz de los resultados por Termoluminiscencia. Manuscrito en posesión del autor.

Campbell, R.

2004 El Trabajo de Metales en la Araucanía (Siglos X-XVII d.C.). Memoria para optar al título de arqueólogo. Universidad de Chile, Santiago. 
2010 Archivo de Fechados Absolutos para Chile Sur. http:// bit.ly/byNd3M. Consultado el 6 de marzo de 2010.

Castro, V. y L. Adán

2001 Abriendo diálogos. Una mirada entre la etnohistoria y la arqueología del área Centro-Sur de Chile: asentamientos en la zona Mapuche. Revista Werken 2:5-35.

Carabias, D., M. Chapanoff y L. Adán

2007 Evidencias de navegación en ambientes lacustres precordilleranos andinos: evaluación arqueológica subacuática del sitio "Dos canoas del Lago Calafquén". En Arqueología de Fuego-Patagonia. Levantando Piedras, Desenterrando Huesos... y Develando Arcano, editado por F. Morello, M. Martinic, A. Prieto y G. Bahamonde, pp. 503-514. Ediciones CEQUA, Punta Arenas.

Catalán, R.

1999 Caracterización de la vegetación de la cuenca del lago Calafquén y sus potencialidades para la ocupación humana. Informe de Avance Proyecto Fondecyt 1970105. Manuscrito en posesión del autor.

Cordero, R.

2010 Organización tecnológica y variabilidad funcional del conjunto lítico del sitio Los Resfalines-1. Actas del XVII Congreso Nacional de Arqueología, Tomo 1, pp. 515-522. Sociedad Chilena de Arqueología, Universidad Austral de Chile. Ediciones Kultrún, Valdivia.

Correa, I. y F. Bahamondes

2009 Alfarería de Tierras Altas parte II: Informe de Análisis Cerámico aleros Cabeza de Indio-1 y Purranque 3. Informe de Avance Proyecto Fondecyt 1060216. Manuscrito en posesión de los autores.

Crumley, C.

1995 Heterarchy and the analysis of Complex Societies. En Heterarchy and the Analysis of Complex Societies. Archaeological Papers of the American Anthropological Association 6, pp. 1-5. Washington.

2007 Notes on a new paradigm. En Socialising Complexity. Structure, Interaction and Power in Archaeological Discourse, editado por S. Kohring y S. Wynne-Jones, pp. 30-36. Oxbow Books, Oxford.

Dillehay, T.

1990 Araucanía. Presente y Pasado. Editorial Andrés Bello, Santiago.

2007 Monuments, Empires and Resistance. The Araucanian Polity and Ritual Narratives. Cambridge University Press, New York

Dillehay, T., M. Pino, R. Bonzani, C. Silva, J. Wallner y C. Le Quesne

2007 Cultivated wetlands and emerging complexity in southcentral Chile and long distance effects of climate change. Antiquity 81:949-960.

Donoso, S. y M.J. Gallego

2008 Informe de análisis cerámico sitios Flor del Lago-1, Playa Negra-1, Liucura-1, y Villarrica W8. Informe de Avance Proyecto Fondecyt 1060216. Manuscrito en posesión de los autores.

Falabella, F. y L. Sanhueza

2005-2006. Interpretaciones sobre la organización social de los grupos alfareros tempranos de Chile Central: alcances y perspectivas. Revista Chilena de Antropología 18:105-133.

Flannery, K.

1975 La Evolución Cultural de las Civilizaciones. Editorial Anagrama, Barcelona.
Foerster, H. y H. Gundermann

1996 Religiosidad mapuche contemporánea: elementos introductorios. En Culturas de Chile. Etnografía. Sociedades Indígenas Contemporáneas y su Ideología, editado por J. Hidalgo, V. Schiapacasse, H. Niemeyer, C. Aldunate y P. Mege, pp. 189-240. Editorial Andrés Bello, Santiago.

Fried, M.

1985 Sobre la evolución de la estratificación social y el Estado. En Antropología Política, editado por J. Llovera, pp. 133-151. Editorial Anagrama, México D.F.

García, C.

2005 Estrategias de Movilidad de Cazadores Recolectores durante el Período Arcaico en la Región del Calafquén, Sur de Chile. Memoria para optar al título de arqueólogo. Departamento de Antropología, Facultad de Ciencias Sociales, Universidad de Chile, Santiago.

2006a Análisis de los materiales líticos provenientes de recolección superficial y excavación en la sección cordillera, durante el año 2005. Informe Final Proyecto Fondecyt 1040326. Manuscrito en posesión del autor.

2006b Los artefactos óseos de Marifilo-1. Una aproximación a la tecnología ósea entre los cazadores recolectores de la selva valdiviana. Revista Werken 8:91-100.

2007 Análisis del conjunto lítico proveniente de excavaciones de sondeos en sitios arqueológicos en la provincia de Cautín, IX Región de La Araucanía. Informe de Avance Proyecto Fondecyt 1060216. Manuscrito en posesión del autor.

Godoy, M.

2010 Uso del bosque templado en la cuenca del Valdivia. Una aproximación etnográfica y etnoarqueológica. Actas del XVII Congreso Chileno de Arqueología, Tomo 1, pp. 505-513. Sociedad Chilena de Arqueología, Universidad Austral de Chile. Ediciones Kultrún, Valdivia.

Grebe, M.E., S. Pacheco y J. Segura

1972 Cosmovisión mapuche. Cuadernos de la Realidad Nacional 14:46-73, Santiago.

Hajduk, A

1986 Arqueología del Montículo Angostura. Primer Fechado Radiocarbónico Provincia del Neuquén. Ediciones Culturales Neuquinas. Museo Histórico Provincial, Neuquén.

Hodder, I.

1994 Interpretación en Arqueología. Corrientes Actuales. Editorial Crítica, Barcelona.

Inostroza, J. y M. Sánchez

1984 Salvataje de cerámica arqueológica en el Liceo Industrial B-22, Temuco, Provincia de Cautín, IX Región. Boletín Museo Regional de la Araucanía 1:96-97.

Johnson, E. y T. Earle

2003 La Evolución de las Sociedades Humanas. Ariel Prehistoria, Barcelona.

Latcham, R.

192 La Organización Social y las Creencias Religiosas de los Araucanos. Imprenta Cervantes, Santiago.

Lehnebach, C., M.E. Solari, L. Adán y R. Mera

2008 Plant macro-remains from a rock shelter in the temperate forests of southern Chile. Vegetation History and Archaeobotany 17:403-413.

McGuire, R.

1983 Breaking down cultural complexity: Inequality and heterogeneity. En Advances in Archaeological Method and Theory Vol. 6, editado por M.B. Schiffer, pp. 91-142. Academic Press, New York. 
Menghin, O.

1962 Estudios de Prehistoria Araucana. Acta Prehistórica III-IV:49-101. Buenos Aires.

Mera, R.

2008 Análisis lítico del sitio Flor del Lago-1. Informe de Avance Proyecto Fondecyt 1060216. Manuscrito en posesión del autor.

Mera, R. y D. Munita

2008 Informe Ejecutivo Salvataje Sitio "Villa JMC-01"Labranza. Región de la Araucanía. Consejo de Monumentos Nacionales, Santiago. Manuscrito en posesión de los autores.

2006 Escuela Collico-1, un cementerio Alfarero Temprano en el valle central de la cuenca de Valdivia. Boletín de la Sociedad Chilena de Arqueología 39:51-68.

Mera, R. y M. Becerra

2002 Análisis del material lítico de los sitios de la costa del Calafquén. Museos 25:7-12.

Mera, R., L. Adán, S. Donoso y F. Bahamondes

2010 El alfarero tardío en aleros de la costa norte del lago Calafquén. Actas del XVII Congreso Nacional de Arqueología, Tomo 1, pp. 523-533. Sociedad Chilena de Arqueología, Universidad Austral de Chile. Ediciones Kultrún, Valdivia.

Mera, R., D. Munita y T. Rudloff

2009 Informe mensual de monitoreo arqueológico. Proyecto Mejoramiento Ruta S-69, sector Pedregoso-Villarrica, IX Región. Período junio-julio 2009. Manuscrito en posesión de los autores.

Mera, R. y L. Adán

2000 Comunicación de nuevos sitios Pitrén a partir del estudio de colecciones. Actas del XIV Congreso Nacional de Arqueología, 345-367. Museo Regional de Atacama, Sociedad Chilena de Arqueología, Copiapó.

Munita, D. y L. Adán

2009 Prospecciones arqueológicas en el eje volcánico Villarrica-Lanín (centro sur de Chile). Aplicación de nuevas estrategias metodológicas y resultados. Arqueometría Latinoamericana: Segundo Congreso Argentino y Primero Latinoamericano, editado por O. Palacios, C. Vásquez, T. Palacios y E. Cabanillas. Vol. 2, pp. 508-514. Comisión Nacional de Energía Atómica, Buenos Aires.

Navarro, X.

2004 Paisajes arqueológicos y territorialidad en la zona Centro Sur de Chile. Recuento actualizado de la historia prehispánica del área ubicada entre Tirúa y Valdivia. Cultura, Hombre y Sociedad 8:71-86.

Navarro, X. y M. Pino

1999 Estrategias adaptativas en ambientes costeros del bosque templado lluvioso de la zona mapuche. Una reflexión desde el precerámico. Actas de las III Jornadas de Arqueología de la Patagonia, pp. 65-82. INAPL y Universidad del Comahue, Neuquén, Buenos Aires.

Ocampo, C., R. Mera y P. Rivas

2004 Cementerios Pitrén en el By Pass de Temuco. Actas del IV Congreso Chileno de Antropología, Tomo II, pp. 14621475. Colegio de Antropólogos de Chile, Universidad de Chile, Santiago.

Pino, M., L. Adán y O. Seguel

2004 Geoarcheology of the Area of Calafquén lake, Southwestern flank of Villarrica Volcano. En Villarrica Volcano $\left(39.5^{\circ} \mathrm{S}\right)$, Southern Andes, Chile, editado por
L. Lara y J. Clavero. Servicio Nacional de Geología y Minería, Boletín 61:61-67.

Politis, G.

2004 Tendencias de la etnoarqueología en América Latina. Teoría Arqueológica en América Latina. Serie Teórica 3:85-117. Universidad Nacional de la Provincia de Buenos Aires, Buenos Aires.

Quiroz, D.

2010 Ocupaciones El Vergel en las costas septentrionales de La Araucanía: Una secuencia cronológica por termoluminiscencia. Actas del XVII Congreso Nacional de Arqueología, Tomo 1, pp. 441-450. Sociedad Chilena de Arqueología, Universidad Austral de Chile. Ediciones Kultrún, Valdivia.

1997 Fragmentos recuperados: un breve panorama histórico para la Isla Mocha. En La Isla de las Palabras Rotas, compilado por D. Quiroz y M. Sánchez, pp. 237-241. Ediciones de la Biblioteca Nacional de Chile. Centro de Investigaciones Diego Barros Arana, Santiago.

Quiroz, D. y M. Sánchez

2005 La secuencia Pitrén-El Vergel en Isla Mocha: soluciones de continuidad y distinciones culturales. Actas del XVI Congreso Nacional de Arqueología, pp. 369-378. Museo de Historia Natural de Concepción y Sociedad Chilena de Arqueología, Tomé-Concepción.

Quiroz, D., Vásquez, M. y M. Sánchez

1997 Quino-1, Un sitio alfarero temprano en la región centrosur: noticia y comentario para un fechado. Boletín Sociedad Chilena de Arqueología 24:49-52.

Reyes, V., L. Sanhueza, y L. Adán

2003-2004 Alfarería doméstica y funeraria de la región del Calafquén. Revista Chilena de Antropología 17:151-179.

Reyes, V.

2008 Microvariaciones en las cadenas operativas de producción cerámica durante el período alfarero tardío del área lacustre de los Andes occidentales, Latitud 39S, Patagonia Septentrional. En Arqueología de Patagonia: una mirada desde el último confin, Actas de las VII Jornadas de Arqueología de la Patagonia, editado por M. Salemme, F. Santiago, M. Alvarez, E. Piana, M. Vázquez y E. Mansur, Editorial Utopías, Ushuaia, en prensa.

Rosales, D. de

1989 [1674] Historia General del Reino de Chile. Flandes Indiano. Tomo I. Editorial Andrés Bello, Santiago.

Sahlins, M.

1981 Las Sociedades Tribales. Nueva Colección Labor, Barcelona.

Sánchez, M.

1997 El período alfarero en la Isla Mocha. La Isla de las Palabras Rotas, compilado por D. Quiroz y M. Sánchez, pp. 103-131. Ediciones de la Biblioteca Nacional de Chile. Centro de Investigaciones Diego Barros Arana, Santiago.

Sánchez, M., J. Inostroza y P. Sanzana

1981-82 Informe preliminar de la excavación de un cementerio arqueológico en el Campus Andres Bello. Anales Universidad de La Frontera 1981-82:171-181.

Sánchez, M. y J. Inostroza

1984 Hallazgo de urnas funerarias en la población Quinta Santa Elvira, Temuco, Provincia de Cautín, IX Región. Boletín Museo Regional de la Araucanía 1:95-96.

Sanhueza, L. y F. Falabella

1999-2000 Las comunidades alfareras iniciales en Chile Central. Revista Chilena de Antropología 15:29-47. 
Silva, O.

1984 En torno a la estructura social de los mapuche prehispánicos. Cultura, Hombre y Sociedad 1:89-115.

Souvatzi, S.

2007 Social complexity is not the same as hierarchy. En Socialising Complexity. Structure, Interaction and Power in Archaeological Discourse, editado por S. Kohring y S. Wynne-Jones, pp. 37-59. Oxbow Books, Oxford.

Stern, Ch., C. García, X. Navarro y J. Muñoz

2009 Sources and Distribution of Different Obsidian Types from Archaeological Sites in Central-South Chile. Magallania 37:19-38.

Valdés, C., M. Sánchez, J. Inostroza, P. Sanzana y X. Navarro 1982 Excavaciones arqueológicas en el Alero Quillén 1, Provincia Cautín, Chile. Actas del IX Congreso Nacional de Arqueología Chilena, pp. 399-435. Museo de La Serena, Sociedad Chilena de Arqueología. La Serena.

Velásquez, H. y L. Adán

2004 Marifilo 1: evidencias arqueofaunísticas para entender las relaciones hombre y bosques templados en los sistemas lacustres precordilleranos del Centro Sur de Chile. En Contra Viento y Marea. Arqueología de la Patagonia, editado por M. Civalero, P. Fernández y A. Guraieb, pp. 507-520. Instituto Nacional de Antropología y Pensamiento Latinoamericano y Sociedad Argentina de Antropología, Buenos Aires.

Wynne-Jones S. y S. Kohring

2007 Socialising complexity. En Socialising Complexity. Structure, Interaction and Power in Archaeological Discourse, editado por S. Kohring y S. Wynne-Jones, pp. 2-28. Oxbow Books, Oxford.

\section{Notas}

1 La secuencia cronológica propuesta por Menghin (1962) constituyó la primera periodificación sistemática y basada en trabajos arqueológicos de campo. Su propuesta adhiere a nociones de historia cultural con un fuerte componente difusionista y un énfasis en la filogenia cultural de los araucanos. De acuerdo al autor, “...la etnohistoria del país araucano nos ofrece, curiosamente, dos puntos fijos: uno al comienzo, con los antepasados de los indios canoeros, y tal vez de los Pehuenches; el otro al final con los Araucanos históricos y protohistóricos. Serán necesarios intensivos trabajos de campo, ante todo exactas excavaciones estratigráficas y de cementerios, para dilucidar el tiempo intermedio" (Menghin 1962:52).

2 Es Aldunate (1989:335) quien define explícitamente la acepción en que usa el concepto de complejo funerario como "todos los elementos del comportamiento social que se reflejen en el registro arqueológico de los sitios funerarios en un lugar y tiempo determinados (cfr. Lanning 1967:209). Cada complejo puede presentar fases locales o cronológicas (cfr. Pollard 1970:38)".

3 En el caso de la zona lacustre subandina, los estudios se han desarrollado en el marco de un programa de investigación iniciado hace más de diez años financiado con fondos públicos (proyectos FONDECYT), permitiendo la identificación de un conjunto variado de sitios arqueológicos, mientras que en el Cautín, la mayor parte de los antecedentes disponibles provienen de rescates de sitios de cementerio impactados por el crecimiento urbano, en el marco de la legislación nacional ambiental.

4 Dicho ejercicio se fundamenta en la utilidad que la reflexión comparativa o analógica ha prestado a la reconstrucción arqueológica, inherente a ella. Sin lugar a dudas, los resultados de un enfoque comparativo están relacionados con la posición teórica del estudio y el investigador y, evidentemente, el esfuerzo que acá presentamos se distancia del más conocido cross cultural approach (cfr. Peregrine 2001, 2004) y se acerca a lo propuesto por autores como Hodder (1993) y Spencer (1992) sobre las amplias posibilidades que ofrece la comparación para comprender la variabilidad o diferencias. Añadimos, como estos últimos autores, la apremiante necesidad del vínculo teórico con la historia, en este caso con la historia comparada, rescatando aquellas perturbadoras "particularidades" a los ojos del clásico cross cultural approach. Veáse por ejemplo el estudio sobre las elites del siglo XVII en Venecia y Amsterdam (Burke 1996).

5 Corresponde al cementerio de Challupén excavado por Mauricio van de Maele, distinto al excavado por Berdichewsky y Calvo.

6 Consideramos estas materias primas como alóctonas, pues si bien claramente son propias del ámbito cordillerano, las hemos registrado en sectores más septentrionales: faldeos y valles que bajan al norte del volcán Sollipulli y en el valle del Alto Bío-Bío, al sur de Lonquimay.

7 La datación fue hecha directamente sobre el maíz.

8 Debe notarse que la fecha de 10 d.C. corresponde al sitio P-25-1, en la Isla Mocha. Se trata de un sitio habitacional y no a un cementerio propiamente tal, pese a que en el depósito se encontró el enterratorio de un individuo subadulto cuya correspondencia con los niveles tempranos no es certera (Quiroz y Sánchez 1997).

9 Corresponden a muestras de carbón y cerámica. Para una exhaustiva recopilación de dataciones absolutas de la zona véase Campbell 2010.

10 Hipótesis que añade además una probable continuidad entre Pitrén y Mapuche en la zona septentrional, meridional y oriental (Aldunate 1989:348, Figura 7).

11 Complejizando más el asunto, notemos que la variedad ollas con estriamiento anular fue tradicionalmente entendida como parte del conjunto cerámico del Complejo El Vergel y Mapuche (Aldunate 1989), sin embargo, recientes análisis de esta modalidad, también denominada acanalada o corrugada, señalan una posición cronológica más temprana y una mayor profusión en sitios cordilleranos como el alero Cabeza de Indio-1 en Melipeuco (Correa y Bahamondes 2009).

12 Tal como puntualizan las autoras, la situación descrita en el Calafquén en fechas tardías no es directamente ampliable a lagos vecinos como el Villarrica que constituiría una prolongación del Toltén como frontera meridional de la dispersión de cerámica Vergel, así como un lugar privilegiado en una ruta de movilidad transversal.

13 El surgimiento de El Vergel y de algunas de sus tecnologías en la zona es recurrentemente interpretada como resultado de un proceso de andinización y del contacto con poblaciones 
septentrionales, a la vez que se destaca la continuidad con los momentos previos y una perspectiva que enfatiza el desarrollo local (p. ej., Aldunate 2005:334-335; Campbell 2004:95-96; Dillehay et al. 2007:956, 958). Resulta evidente, en consecuencia, la necesidad de desarrollar investigaciones acotadas a diferentes localidades que permitan profundizar y particularizar el estado de la cuestión.

14 Las determinaciones de los tipos de pasta se han practicado mayoritariamente sobre cerámica fragmentada (Reyes et al. 2003-2004).

15 Dichos términos se emplean en el sentido otorgado por Bourdieu $(1985,1999)$ reinterpretados para contextos prehispánicos. Campo o espacio social "can be represented as a space (with several dimensions) constructed on the basis of principles or differentiation or distribution constitued by the set of properties active within the social universe in question, i.e. capable of conferring strenght, power within that universe, on their holder. Agents and groups of agents are tus defined by their relative positions within this space" (Bourdieu 1985:196). En este espacio social se participa distintivamente de lo económico y lo cultural (capital económico y cultural) (Bourdieu 1997:17), por medio de disposiciones o habitus. De acuerdo al autor "el espacio de las posiciones sociales se retraduce en un espacio de tomas de posición a través del espacio de las disposiciones (o de los habitus)... a toda clase de posición corresponde una clase de habitus (o de aficiones) producidas por los condicionamientos sociales asociados a la condición correspondiente y, a través de estos habitus y de sus capacidades generativas, un conjunto sistematico de bienes y de propiedades, unidos entre sí por una afinidad de estilo" (Bourdieu 1999:19).

16 Literatura antropológica aplicada a la comprensión de la estructura social de la población mapuche histórica temprana ha planteado que estas corresponderían a sociedades tribales al decir de Sahlins, en los que se reconocerían linajes, clanes y familias (Silva 1984: 93-94). Se ha planteado también que ellas pueden ser abordadas en cuanto sociedades segmentarias. En éstas se reconocen sociedades con descendencia unilineal cuyos linajes están en continuo proceso de segmentación social y territorial y oposición complementaria, sin perder su unidad corporativa, mientras que en otras ocurre la repetición de sistemas pequeños y autosuficientes, por medio de procesos de fisión en el cual el primer grupo desaparece y quedan dos o más grupos iguales (veáse Bechis 1989 para una extensa discusión sobre el uso de los conceptos segmentarios y segmental). En este mismo sentido se entendería la distinción que hace Sahlins entre esquemas tribales piramidales y aquellos esquemas tribales segmentales en que los grupos locales menores no se integran en un esquema político mayor y estable (Sahlins 1981).

17 Estos antecedentes históricos se integran de manera referencial y desde el punto de vista de la utilidad de la reflexión analógica o de paralelos etnográficos para la interpretación arqueológica (Hodder 1990; Politis 2004). Evidentemente, no suponemos que exista una continuidad histórica absoluta que permita la aplicación del método histórico directo entre lo que se define arqueológicamente como Pitrén y la población mapuche histórica temprana. Esta perspectiva ha hecho posible interesantes reconstrucciones en la historia prehispánica de la zona central y centro-sur de Chile que asumen los riesgos y dificultades inherentes a estas aproximaciones (veáse, entre otros, Castro y Adán 2001; Dillehay 2007; Sanhueza y Falabella 1999-2000).

18 Para momentos históricos se ha destacado el vínculo de los volcanes con los antepasados, reflejado en la figura del Pillán (Rosales 1989: 155, 189-190), como asimismo la relación de los cerros con el mito fundacional de Trentren y Caicai (Rosales 1989: 27-29). El recorrido de estas creencias, tempranamente documentadas por Rosales, ha sido retomado en estudios históricos y antropológicos centrados en diferentes temáticas y orientaciones teóricas (entre otros, Boccara 2007; Dillehay 2007; Foerster y Gundermann 1996; Grebe 1972; Latcham 1924). Como destacamos en la nota previa no proyectamos hacia el pasado el sistema de creencias de las poblaciones mapuche históricas, aunque hacemos notar un evidente vínculo formal o "analógico" en la disposición a seleccionar lugares altos para dar sepultura a sus difuntos y en la mirada de éstos hacia el Este. Se enfatiza además la existencia de este patrón con anterioridad a los momentos alfarero tardíos. En el Calafquén, los impactos del volcanismo en el asentamiento han sido tratados para el caso del sitio Marifilo-1 (Pino et al. 2004), mientras que desde una perspectiva fenomenológica se ha explorado la constitución de topos referenciales y significativos constituidos por el eje dewiñ/volcán y lafken/lago en las formas de habitar de las poblaciones prehispánicas (Alvarado 2000).

19 El tratamiento y definición de la complejidad social en la discusión arqueológica ha generado un copioso debate fundamentalmente en literatura de habla inglesa que actualiza modelos teóricos desarrollados en la antropología desde la década de los 50, aproximadamente. En efecto, para algunos la complejidad social es entendida como una categoría o nivel de organización sociopolítica jerárquica y desigual, la cual ha sido abordada generalmente desde los estudios de evolución social (p.ej., Fried 1967; Sahlins1981; entre otros). En la visión de esta perspectiva "que la evolución sociocultural se haya producido -o no- ha dejado de ser un problema a dilucidar. El trabajo arqueológico procedente de todos los continentes documenta cambios desde tempranas sociedades a pequeña escala hacia otras complejas más tardías" (Johnson y Earle 2003:12). En cambio, como WynneJones y Kohring (2007) han puntualizado, la complejidad -esta vez con c minúscula- puede ser entendida como una herramienta conceptual para comprender la integración e interacción social, bajo diferentes enfoques como la agencia, el habitus, la acción performática, entre otros. Como resulta evidente, en consecuencia, el área de los estudios de complejidad social, congrega un conjunto de perspectivas analíticas, algunas evidentemente neoevolucionistas y otras de filiación posestructural por lo cual es necesario hacer explícitas las variables de análisis que se manejarán.

20 Perspectivas que han entrampado la discusión en torno al tipo de organización social de los tempranos mapuche, entre quienes adhieren a dichas categorías y aquellos renuentes a usarlas (cfr. Boccara 2007; Silva 1984).

21 Esta aparece sólo en algunos individuos de sexo masculino del conjunto analizado (Aspillaga y Retamal en Ocampo et al. 2001).

22 El papel de las dinámicas heterárquicas es aplicado por Dillehay (2007) en su análisis de la estructura política y religiosa de los tempranos mapuche.

23 Usamos el concepto como es actualizado para el caso mapuche por Dillehay (2007:73-75). 
